FAST 704 MHz FERROELECTRIC TUNER FOR SUPERCONDUCTING CAVITIES

\title{
Omega-P, Inc.
}

291 Whitney Avenue, Suite 401

New Haven, CT 06511

\section{FAST 704 MHz FERROELECTRIC TUNER FOR SUPERCONDUCTING CAVITIES}

Final Report on Phase I DoE SBIR grant DE-SC000 6229

Topic 44a: Nuclear Physics Accelerator Technology

Materials and Components for Radio Frequency Devices

J. L. Hirshfield, Principal Investigator

tel: (203) 789-1164, e-mail: jay@ omega-p.com

\section{CONTENTS}

I. Executive Summary

II. Identification and Significance of the Problem or Opportunity, and Technical Approach

IIa. Introduction

IIb. Technical approach

III. Degree to which Phase I has Demonstrated Technical Feasibility 8

IIIa. Technical objectives $\quad 8$

IIIb. General scheme for tuner 9

IIIc. Conceptual design for modular coaxial phase shifter 11

IIId. Engineering design for modular coaxial phase shifter $\quad 15$

IIIe. Measurements of the dielectric constant and loss tangent of metal-coated ferroelectric samples 22

References

Relevant Publications and Patents

Material in this Report was developed by Omega-P senior consultants Dr. Sergey Yu. Kazakov and Dr. Vlacheslav P. Yakovlev; and by Dr. Sergey Shchelkunov who worked under a sub-grant from Omega-P to Yale University. Dr. Hirshfield, in his role as Principal Investigator, coordinated activities of the participants and edited the Report.

NO DISTRIBUTION LIMITS APPLY TO THIS REPORT

April 12, 2012 
Omega-P, Inc.

Final Report on DoE 2011 Phase I grant DE-SC0006229

Topic 44a: Nuclear Physics Accelerator Technology, Materials and Components for Radio Frequency Devices

FAST 704 MHz FERROELECTRIC TUNER FOR SUPERCONDUCTING CAVITIES

\section{EXECUTIVE SUMMARY}

The Omega-P SBIR project described in this Report has as its goal the development, test, and evaluation of a fast electrically-controlled L-band tuner for BNL Energy Recovery Linac (ERL) in the Electron Ion Collider (EIC) upgrade of the Relativistic Heavy Ion Collider (RHIC) at Brookhaven National Laboratory (BNL). The tuner, that employs an electrically-controlled ferroelectric component, is to allow fast compensation to cavity resonance changes. In ERLs, there are several factors which significantly affect the amount of power required from the wallplug to provide the RF-power level necessary for the operation. When beam loading is small, the power requirements are determined by $(i)$ ohmic losses in cavity walls, (ii) fluctuations in amplitude and/or phase for beam currents, and (iii) microphonics. These factors typically require a substantial change in the coupling between the cavity and the feeding line, which results in an intentional broadening of the cavity bandwidth, which in turn demands a significant amount of additional RF power. If beam loading is not small, there is a variety of beam-drive phase instabilities to be managed, and microphonics will still remain an issue, so there remain requirements for additional power. Moreover ERL performance is sensitive to changes in beam arrival time, since any such change is equivalent to phase instability with its vigorous demands for additional power.

In this Report, we describe the new modular coaxial tuner, with specifications suitable for the $704 \mathrm{MHz}$ ERL application. The device would allow changing the RF-coupling during the cavity filling process in order to effect significant RF power savings, and also will provide rapid compensation for beam imbalance and allow for fast stabilization against phase fluctuations caused by microphonics, beam-driven instabilities, etc. The tuner is predicted to allow a reduction of about ten times in the required power from the RF source, as compared to a compensation system with narrower bandwidth. It is planned to build a $704 \mathrm{MHz}$ version of the tuner, to check its underlying principles, and to make high-power tests at power densities aimed towards controlling $50 \mathrm{~kW}$ of average power. Steps towards this goal will be limited by, among other factors, losses in the actual ferroelectric elements in the ferroelectric assemblies. As the ferroelectric material loss tangent is reduced through efforts by the supplier Euclid TechLabs LLC, the concomitant power loss in its ferroelectric assemblies will drop, and the average power-handling capability of the Omega-P tuner will rise. It can thus be anticipated that the Phase II development project of the $704 \mathrm{MHz}$ tuner will be iterative, but the pace and ultimate power-handling level of the tuner is difficult to predict at this early stage in Euclid's development program. Fortunately, since Omega-P's conceptual tuner is a simple module (nominally rated for $5 \mathrm{~kW}$ ), so that the number of modules required in each tuner can be chosen, depending upon the cavity power level needed, plus the power for tuner losses. 


\section{IDENTIFICATION AND SIGNIFICANCE OF THE PROBLEM OR OPPORTUNITY, AND TECHNICAL APPROACH}

\section{Introduction}

Under Topic 44a in the DoE 2010 SBIR Program Solicitation, Phase I proposals were sought to improve or advance superconducting and room-temperature materials or components for RF devices used in particle accelerators. Areas of interest included "...fast ferroelectric microwave components that control reactive power for fast tuning of cavities or fast control of input power coupling." Preliminary results on this subject were developed by Omega-P under its prior DoE 2010 Phase I SBIR grant DE-SC000 0922, entitled "Fast Ferroelectric L-Band Tuner for Superconducting Cavities." That project did not advance into Phase II. The overall goal of the 2011 follow-on Phase I R\&D program reported here was to refine the concept and develop a preliminary engineering design for a new configuration for a $704 \mathrm{MHz}$ ferroelectric phase shifter, namely a modular compact coaxial structure. Plans for the future include designing, building, and testing a $704 \mathrm{MHz}$ fast electrically-driven ferroelectric tuner prototype for controlling the external coupling to RF cavities for the superconducting Energy Recovery Linac (ERL) in the Electron Ion Collider (EIC) upgrade of the Relativistic Heavy Ion Collider (RHIC) at Brookhaven National Laboratory (BNL).

A fast tuner for superconducting (SC) cavities in the ERL is needed to compensate for rapid cavity resonant frequency detunings that can arise, for example, from microphonics and from variations in beam loading. Before immersing readers in technical details of the new coaxial configuration for the $704 \mathrm{MHz}$ ferroelectric tuner for the BNL ERL, it may help to first explain the benefits from such a fast tuner in quantitative terms. One may consider three possible situations. In the first situation, an over-coupled SC cavity is connected to a feeding line directly, as in Fig. 1.

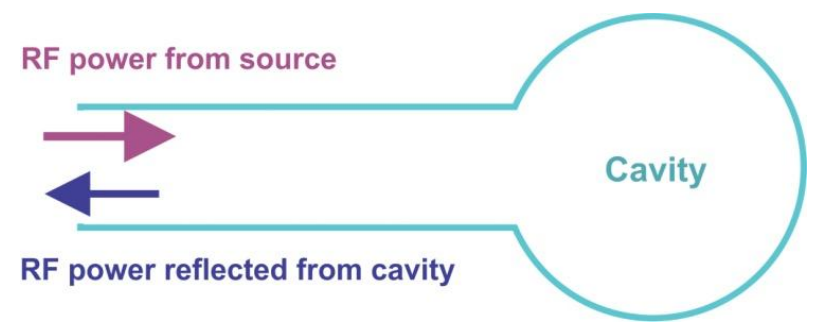

Fig. 1. An over-coupled cavity connected directly to the RF source.

Most of the power is reflected from the cavity.

The coupling in this case is high enough to broaden the cavity bandwidth so as to exceed the range of rapid microphonics detuning of the cavity. This scheme requires more RF power than would be needed if critical coupling could be used, because of high reflections from overcoupling. However, these reflections may be compensated for if a fast tuner is installed between the SC cavity and the RF source, as shown in Fig 2. This allows the coupling value to be constantly adjusted to equal unity, which thus eliminates reflections. 


\section{Omega-P, Inc.}

Final Report on DoE 2011 Phase I grant DE-SC0006229

Topic 44a: Nuclear Physics Accelerator Technology, Materials and Components for Radio Frequency Devices

FAST 704 MHz FERROELECTRIC TUNER FOR SUPERCONDUCTING CAVITIES

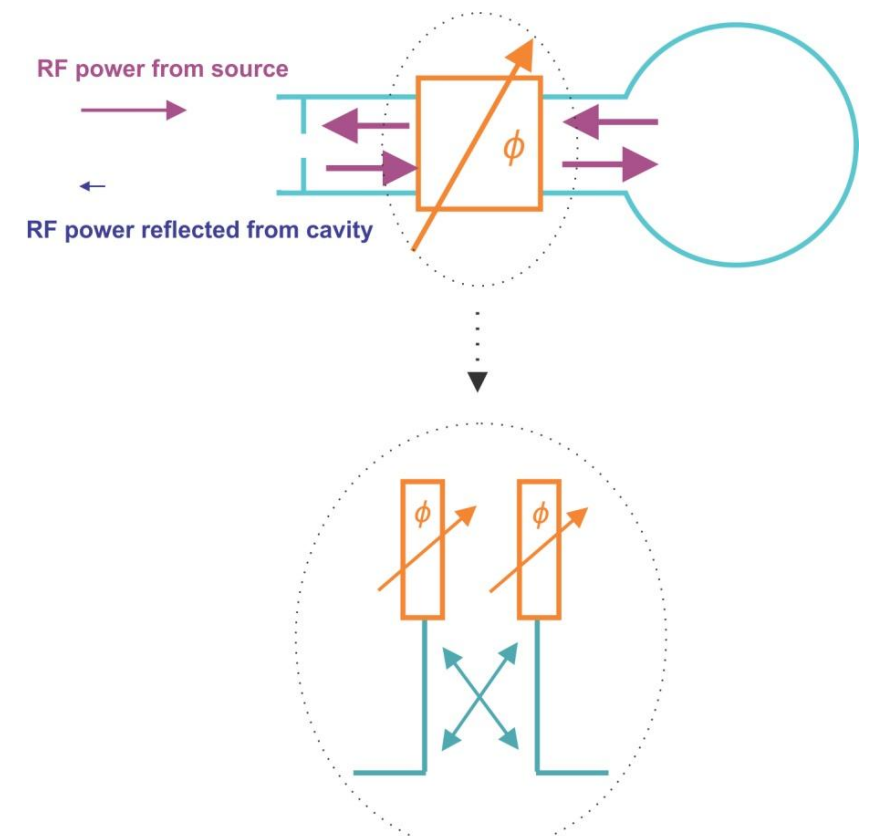

Fig. 2. Superconducting cavity fed through a fast tuner. Power from the RF source compensates only the losses in the tuner, since for this simplified illustration beam loaded is neglected.

The tuner may be built using two reflecting phase shifter and 3-dB hybrid.

The direct coupling to the cavity is achieved using a coupling iris, or diaphragm. The tuner, placed between the diaphragm and the cavity itself, changes the electric length of the line between the diaphragm and the cavity, thereby preserving the cavity resonance frequency during mechanical perturbations due to microphonics. In this way, the cavity coupling to the feeding line is the same as if the cavity were coupled to the RF source as in the first scheme (Fig. 1). Net power flow from the tuner to the cavity and back in the second scheme is to be the same as that from the RF source to the cavity and back in the first scheme. But the power needed is only that necessary to compensate for losses in the phase shifter. (In this simplified explanation, beam loading in the SC cavity is neglected.) This implies, for example, that if the losses in the tuner are $5-10 \%$, then power from the RF source in the second scheme may be reduced by 10-20 times, as compared to power needed from the source in the first scheme. Such a reduction in required $\mathrm{RF}$ power for each SC cavity in a large accelerator would amount to a considerable savings in cost and complexity.

The third scheme, as shown in Fig. 3, embodies a fast phase shifter connected to a dedicated second port of the SC cavity. Efficiency of this scheme is equal to the efficiency of the second scheme. This arrangement requires only one reflecting phase shifter, rather than two (as in Fig. 2), but has the disadvantage of requiring a second port in the SC cavity. A comprehensive systems study is required to determine if the third scheme is less costly than the second. 


\section{Omega-P, Inc.}

Final Report on DoE 2011 Phase I grant DE-SC0006229

Topic 44a: Nuclear Physics Accelerator Technology, Materials and Components for Radio Frequency Devices

FAST 704 MHz FERROELECTRIC TUNER FOR SUPERCONDUCTING CAVITIES

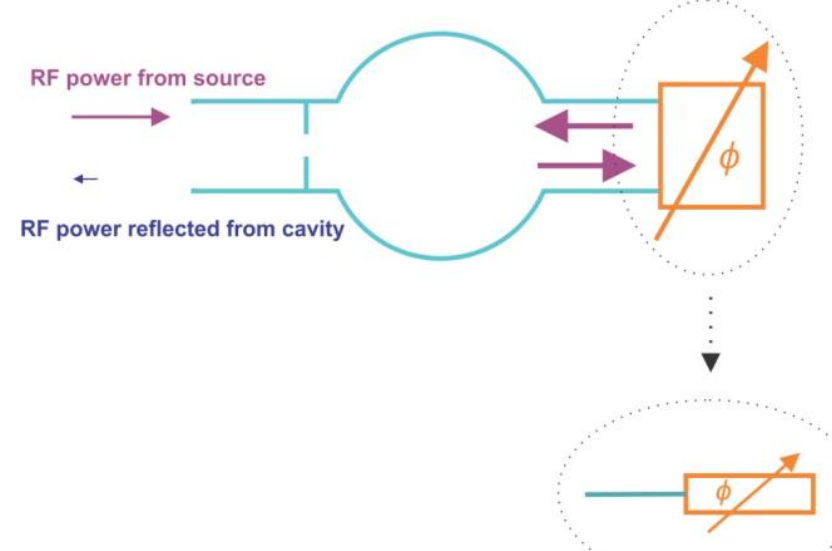

Fig. 3. SC cavity tuning using a separate dedicated port and one reflecting phase shifter.

The power savings ratio (whose reciprocal is the fraction of power needed with a tuner as compared to without) of the second and third schemes as compared to the first is shown in Fig. 4, plotted as a function of the loss tangent of the active ferroelectric material in the fast tuner (to be described below). Losses in metal elements of the structure are also taken into account, as they are not insignificant because of high dielectric constant of the ferroelectric material.

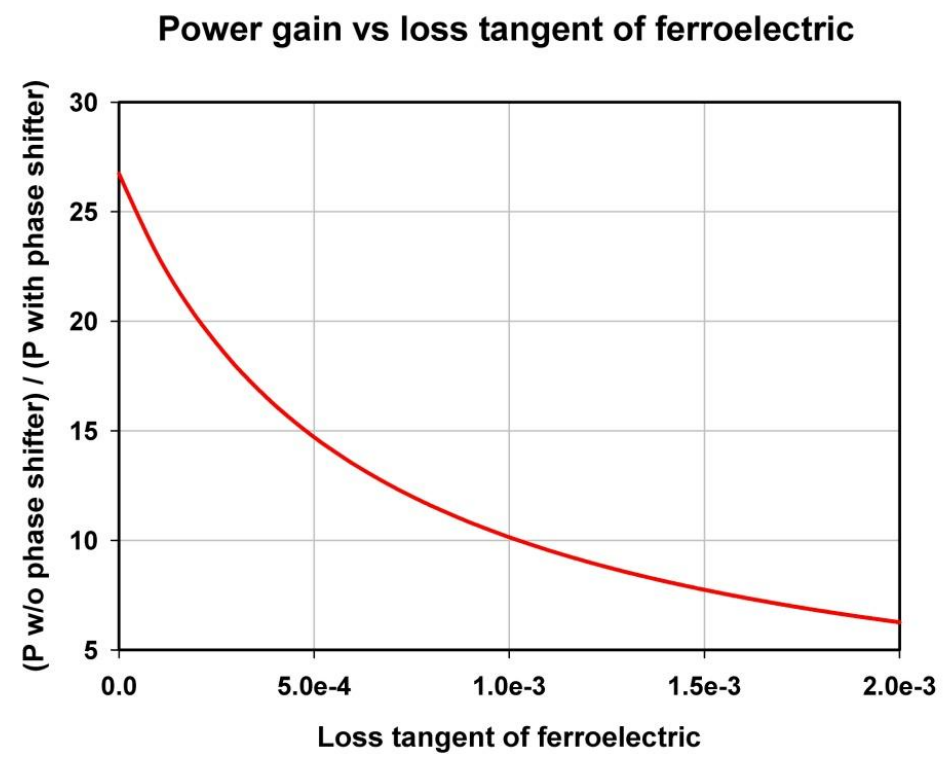

Fig. 4. Efficiency of the scheme with the ferroelectric phase shifter $v s$ ferroelectric loss tangent.

It is the potentially high power ratios shown in Fig. 4 that provide strong motivation for developing the fast ferroelectric tuner described in this report, since a factor-of-10 or so savings in required RF power could represent an enormous cost savings for a full-scale ERL containing many tens of SC cavities. Once proven at $704 \mathrm{MHz}$ for the BNL ERL, it is expected that the same concept can be extended to fast tuners for a wide range of other accelerators, providing a strong market for future commercialization of results of this SBIR project. 


\section{Omega-P, Inc.}

Final Report on DoE 2011 Phase I grant DE-SC0006229

Topic 44a: Nuclear Physics Accelerator Technology, Materials and Components for Radio Frequency Devices

FAST 704 MHz FERROELECTRIC TUNER FOR SUPERCONDUCTING CAVITIES

\section{Technical Approach}

The Omega-P SBIR project described here has as its goal the development, test, and evaluation of a fast electrically-controlled L-band tuner for BNL Energy Recovery Linac (ERL) in the Electron Ion Collider (EIC) upgrade of the Relativistic Heavy Ion Collider (RHIC) at Brookhaven National Laboratory (BNL). The tuner, that employs an electrically-controlled ferroelectric component, is to allow fast compensation to cavity resonance changes. In ERLs, there are several factors which significantly affect the amount of power required from the wallplug to provide the RF-power level necessary for the operation. When beam loading is small, the power requirements are determined by $(i)$ ohmic losses in cavity walls, (ii) fluctuations in amplitude and/or phase for beam currents, and (iii) microphonics. These factors typically require a substantial change in the coupling between the cavity and the feeding line, which results in an intentional broadening of the cavity bandwidth, which in turn demands a significant amount of additional RF power. If beam loading is not small, there is a variety of beam-drive phase instabilities to be managed, and microphonics will still remain an issue, so there remain requirements for additional power. Moreover ERL performance is sensitive to changes in beam arrival time, since any such change is equivalent to phase instability with its vigorous demands for additional power.

To mitigate demands for the additional power, one may (1) change the cavity geometry to offset detuning caused by phase instabilities and/or microphonics, and/or (2) apply a corrective phase shift to the reflected RF wave that is reintroduced to the cavity so as to cancel phase instabilities [1]. The first strategy is accomplished by internal or external motors, or fast internal mechanical piezoelectric (or magnetostrictive) tuners [2,3]. The second approach utilizes fast ferrite or ferroelectric phase shifters that are external to the cryomodules [4,5]. Piezoelectric and other mechanical tuners require operation at cryogenic temperatures and thus permit only limited access in the event of a failure. Further, piezoelectric devices have mechanical resonances which may interfere with control system performance if their own resonance frequency overlaps with the microphonics excitation to be controlled [1]. It has been reported that the piezo-electric tuners may operate as fast as $\sim 25 \mathrm{kHz}$ [6]; however, it is still unknown if piezo-electric tuners are efficient enough at high frequencies. Ferrite phase shifters $[7,8,9]$ are presently limited in their response time to $\sim 30 \mu \mathrm{s}$, while the required response time may be only a few $\mu \mathrm{s}$. The limitation comes mainly from the eddy currents in the ferrite material [8]. One example is the high-power fast ferrite tuner which is currently being developed at CERN for the Superconducting Proton Linac (SPL) [7] operating at $352 \mathrm{MHz}$. This tuner is designed to provide fast phase and amplitude modulation of the drive signal for individual superconducting cavities. The tuner is based on two fast and compact high-power ferrite phase shifters magnetically biased by external coils. The tuning frequency for this device has an upper cut-off at about $2 \mathrm{kHz}$.

Need for $\mu$ s response time is dictated by the phase and amplitude stability requirements of $\sim 0.06 \mathrm{deg}$ and $\pm 3 \times 10^{-4}$, as cited for the Cornell ERL [10]; requirements are similar for the ERL project at BNL [11]. The gain in the control feedback loop should be high enough, and its bandwidth wide enough, to insure this high degree of stability. This translates to a bandwidth of about $1 \mathrm{MHz}$; similar considerations apply to the BNL ERL. This tight restriction rules out contemporary ferrite tuners with their narrow bandwidth and slow response times. 


\section{Omega-P, Inc.}

Final Report on DoE 2011 Phase I grant DE-SC0006229

Topic 44a: Nuclear Physics Accelerator Technology, Materials and Components for Radio Frequency Devices

FAST 704 MHz FERROELECTRIC TUNER FOR SUPERCONDUCTING CAVITIES

As an alternative, Omega-P here describes a design for a fast electrically-controlled ferroelectric phase shifter for ERL applications that may satisfy the power handling capabilities, and phase/ amplitude stability requirements, with a bandwidth exceeding $1 \mathrm{MHz}$.

It should be noted that a successful fast ferroelectric tuner would find a wide application for superconducting cavity stabilization. For example, it will be very useful in the case when a number of cavities are fed by one RF source, as in the Project X pulse linac [12]. In this case, the tuner will provide independent amplitude and phase control of the field in separate cavities that may be especially important for proton linacs. Application of fast ferroelectric phase shifters was proposed by S. Belomestnykh for stabilization of the SC cavity for the ILC damping Ring [13].

In its Phase I proposal, Omega-P initially described two alternative concepts for a fast electrically-controlled ferroelectric $704 \mathrm{MHz}, 50 \mathrm{~kW}$ phase shifter for ERL applications. These concepts are the triple-decker waveguide tuner, and the radial line or coaxial reflecting tuner. One objective during Phase I was to compare the predicted performance of these two approaches, so as to select the superior design for full development during Phase II. However, during Phase I, issues with each of these arose and a new (much more attractive) concept was invented, namely a modular coaxial concept. This new concept is based on the utilization of one small donut-shaped ferroelectric assembly for each coaxial module, which allow the design to be simpler and more flexible, as compared to the previous designs. Each ferroelectric assembly consists of a metalized ferroelectric ring brazed between two copper cups. The assembly allows separate tuning and testing of each assembly prior to installation. The ferroelectric assembly is to be developed by Omega-P's vendor Euclid Techlabs LLC within the scope of its own SBIR project on advanced materials research for accelerator applications.

In Section III of this Report, we describe the new modular coaxial tuner, with specifications suitable for the $704 \mathrm{MHz}$ ERL application. The device would allow changing the RF-coupling during the cavity filling process in order to effect significant RF power savings, and also will provide rapid compensation for beam imbalance and allow for fast stabilization against phase fluctuations caused by microphonics, beam-driven instabilities, etc. The tuner is predicted to allow a reduction of about ten times in the required power from the RF source, as compared to a compensation system with narrower bandwidth. It will be necessary to build a $704 \mathrm{MHz}$ version of the tuner, to check its underlying principles, and to make high-power tests at power densities aimed towards controlling $50 \mathrm{~kW}$ of average power. Steps towards this goal will be limited by, among other factors, losses in the actual ferroelectric elements in the ferroelectric assemblies. As Euclid, during progress under its own DoE-sponsored SBIR development program, reduces the ferroelectric material loss tangent and the concomitant power loss in its ferroelectric assemblies, the average power-handling capability of the Omega-P tuner will rise. It can thus be anticipated that the Phase II development of the $704 \mathrm{MHz}$ tuner will be iterative, but the pace and ultimate power-handling level of the tuner is difficult to predict at this early stage in Euclid's development program. Fortunately, since Omega-P's conceptual tuner is a simple module (nominally rated for $5 \mathrm{~kW}$ ), so that the number of modules required in each tuner can be chosen, depending upon the cavity power level needed, plus the power for tuner losses. 
FAST 704 MHz FERROELECTRIC TUNER FOR SUPERCONDUCTING CAVITIES

\section{DEGREE TO WHICH PHASE I HAS DEMONSTRATED TECHNICAL FEASIBILITY}

\section{IIIa. Technical Objectives}

The ultimate goal of this project has been to develop a $704 \mathrm{MHz}$ fast tuner for the superconducting cavities in the ERL that are need for the RHIC upgrade. Key objectives are both design and technological. The technical objective of the Phase I program has been to develop a preliminary design of a fast external tuner for coupling of ERL cavities using electricallycontrolled ferroelectric phase shifters. Each tuner should allow rapid changes in the coupling of an ERL cavity with the feed line at an average power level of up to $50 \mathrm{~kW}$ at $704 \mathrm{MHz}$. The tuner design involves detailed analytical studies and numerical simulations of a phase shifter in order to minimize electric fields and power losses in the ferroelectric elements, providing at the same time the required tuning range and speed of RF phase changes.

The two concepts for the phase shifter that were initially considered are:

a) a triple-decker concept, similar to one developed for ILC applications [14], and

b) a radial line reflecting tuner.

However, experience with the triple-decker concept demonstrated issues to be resolved with high-voltage breakdown and brazing of ceramics to copper walls. In addition, the concept required two types of ceramic elements: ferroelectric bars and linear ceramic matching bars. The radial line reflecting phase shifter requires a ferroelectric ring of substantial physical size that appeared to be difficult to sinter, metalize and braze. Thus, in Phase I after detailed analysis of electrodynamic and technological problems of both concepts, these have been downgraded in priority in favor of another concept based on use of one small ferroelectric ring assembly for each phase shifter module. These assemblies can be standardized, and are of relatively small size are expected to be relatively easy to manufacture, individually tuned, cooled, and tested. Furthermore, this new phase shifter design requires no linear ceramic matching elements. The small ring ferroelectric assembly terminates a coaxial line composing a phase shifter module, with each module capable of handling about $5 \mathrm{~kW}$ of average RF power; five such modules are to be connected in parallel using a special coupler to handle $25 \mathrm{~kW}$; and two such assemblies mounted in a magic-T would then be able to control the full $50 \mathrm{~kW}$. This modular concept allows for effective dissipation of RF power losses, relative simplicity of design, and ease of replacement in case of failure of one of the modules.

The ferroelectric assemblies are to be developed and fabricated to Omega-P specifications for the $704 \mathrm{MHz}$ tuner by Euclid TechLabs LLC, with support under their own SBIR grant from DoE that concentrates on development of ferroelectric ceramics and of technological means to incorporate these into brazed assemblies. Clearly, success with OmegaP's tuner depends upon Euclid's success in its development of the ferroelectric material, particularly for material having a dielectric constant reduced from 500-600 to about 300, with a tunability of about $6 \%$ at an electric field of $15 \mathrm{kV} / \mathrm{cm}$, and with a loss tangent of $5 \times 10^{-4}$ at 704 MHz. Fabrication by Euclid of the certified assemblies is also essential, so as to insure that contamination of the ceramic that could arise unintentionally during brazing by others, who may 


\section{FAST 704 MHz FERROELECTRIC TUNER FOR SUPERCONDUCTING CAVITIES}

not have access to Euclid's proprietary processes, will not have altered the critical characteristics of the ferroelectric material itself.

\section{IIIb. General Scheme for Tuner}

A tuner may in general be designed as a combination of two fully-reflecting phase shifters and a 3-dB hybrid, as shown in Fig. 5. This device will in principle provide any desired degree of reflection and any desired phase, independently of one another. Thus, the problem is reduced to the design of a fully-reflecting fast-response, low-loss phase shifter.

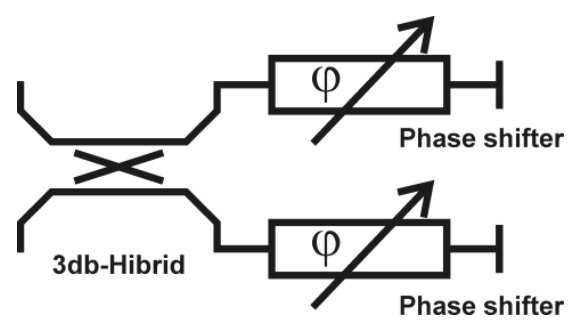

Fig. 5. Combination of 3-dB hybrid and two shorted phase shifters amounts to a tuner.

The proposed overall device configuration is shown in Fig. 6 (see, for example, [5]) that can allow fast electrically-controlled coupling and reflecting phase changes. This device is based on a magic- $T$ with two coaxial phase shifters containing ferroelectric assemblies. Changing the phase shifts of the reflected signal from $0^{\circ}$ to $90^{\circ}$ will change the transmission coefficient from 0 to 1 , or the phase from $0^{\circ}$ to $90^{\circ}$, independently of one another [5]. For a symmetric magic-T,

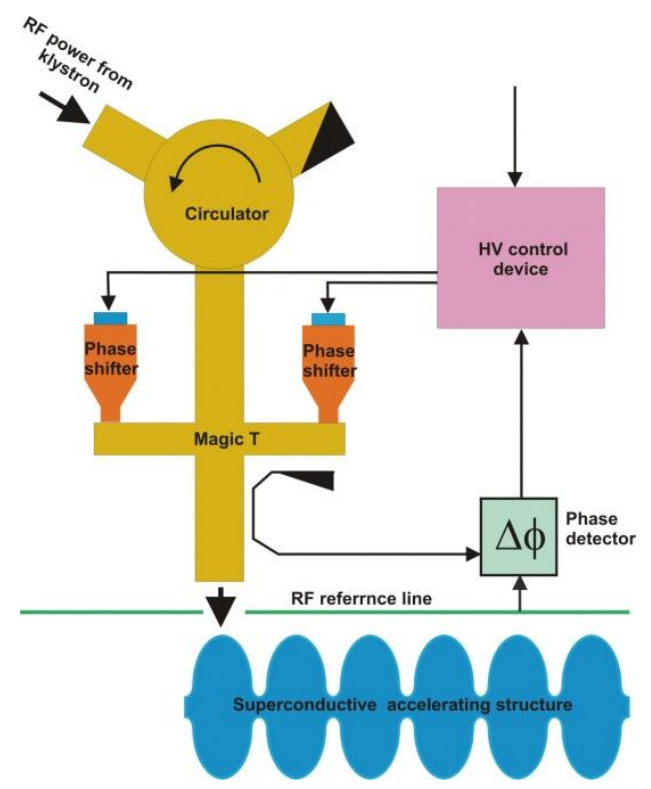

Fig. 6. A possible configuration for SRF cavity phase and amplitude control

Using two external tuners (reflecting phase shifters) and a magic-T. Also shown is a schematic of the feedback loop that would be employed for rapid automated stabilization. 


\section{Omega-P, Inc.}

Final Report on DoE 2011 Phase I grant DE-SC0006229

Topic 44a: Nuclear Physics Accelerator Technology, Materials and Components for Radio Frequency Devices

FAST 704 MHz FERROELECTRIC TUNER FOR SUPERCONDUCTING CAVITIES

the power level at each phase shifter is half of the total power incident in the input port, which thereby reduces the temperature rise in the phase shifter's active element (ferroelectric) that must be accounted for in the design. The phase shifter itself was originally proposed to be designed either as a triple-decker waveguide concept, similar to one considered for ILC applications [14], or a radial line reflecting tuner. Applying bias voltage across the ferroelectric assemblies effects a change in dielectric permittivity of the ferroelectric, which causes a phase advance of the RF wave in the phase shifter, and thus a change in coupling between the cavity and the RF source.

In the new phase shifter concept, one or more ferroelectric assemblies behave as cavities connected as transmission components within waveguides, as shown in Fig. 7. Changing the resonant frequency of each cavity by voltage-tuning the ferroelectric assemblies can change the phase of the transmitted signal while keeping the SWR within an acceptable low range.
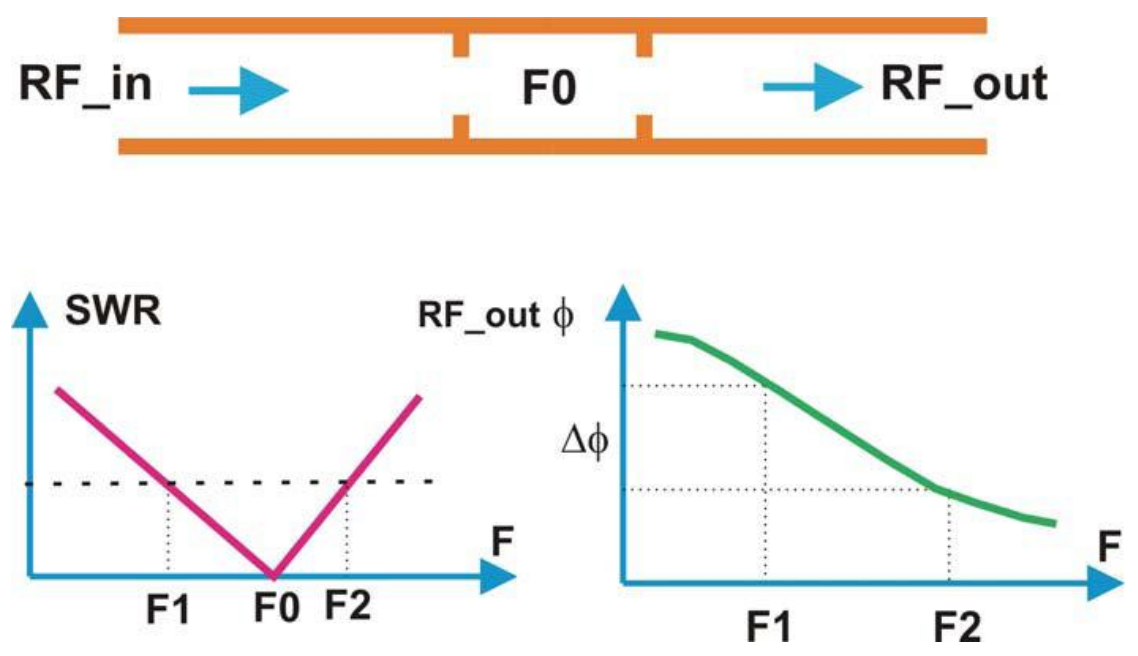

Fig. 7. Equivalent circuit of the strongly coupled ferroelectric phase shifter. The ferroelectric element works as a transmission-type cavity.

In the new concept the ferroelectric assembly is placed inside the transmission line, and the RF energy partially flows past the assembly, as shown in Fig. 8. The coupling is adjusted not by a dielectric matching element, but by selection of the relative sizes of the transmission line and the ferroelectric assembly.

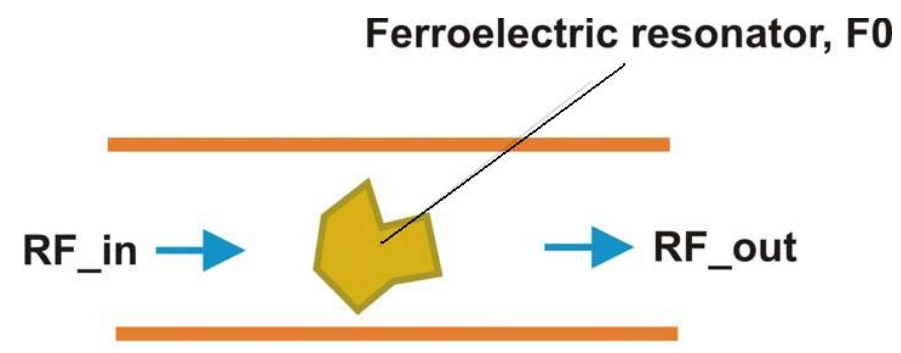

Fig. 8. Sketch of the new concept of the phase shifter. 
Omega-P, Inc.

Final Report on DoE 2011 Phase I grant DE-SC0006229

Topic 44a: Nuclear Physics Accelerator Technology, Materials and Components for Radio Frequency Devices

FAST 704 MHz FERROELECTRIC TUNER FOR SUPERCONDUCTING CAVITIES

\section{IIIc. Conceptual design for modular coaxial phase shifter}

The simplest and most technologically-convenient shape for the ferroelectric element is a pillbox (i.e., a right circular cylinder). However, in order to avoid problems with nearby parasitic modes, it is possible to use a ring (donut) instead of a pillbox. A reference diagram of the ring is shown in Fig. 9. In Fig. 10 the frequencies of the lowest modes of a cylindrical coaxial ring are shown versus its internal radius at fixed frequency of the operating $\mathrm{TM}_{020}$ mode of $704 \mathrm{MHz}$. In this example the ring thickness $h$ is $5 \mathrm{~mm}$, and the dielectric constant $\varepsilon=285$. Stars in Fig. 10 mark possible operating points, which correspond to the cases when the frequency the operating $\mathrm{TM}_{020}$ mode is mid-way between parasitic modes.

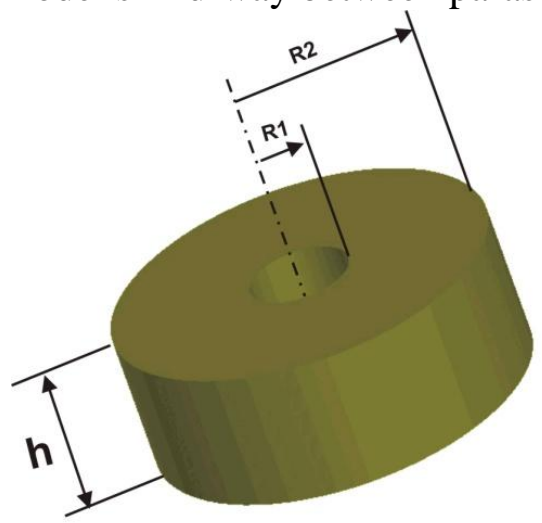

Fig. 9. Reference geometry for a ferroelectric ring, showing dimensional quantities.

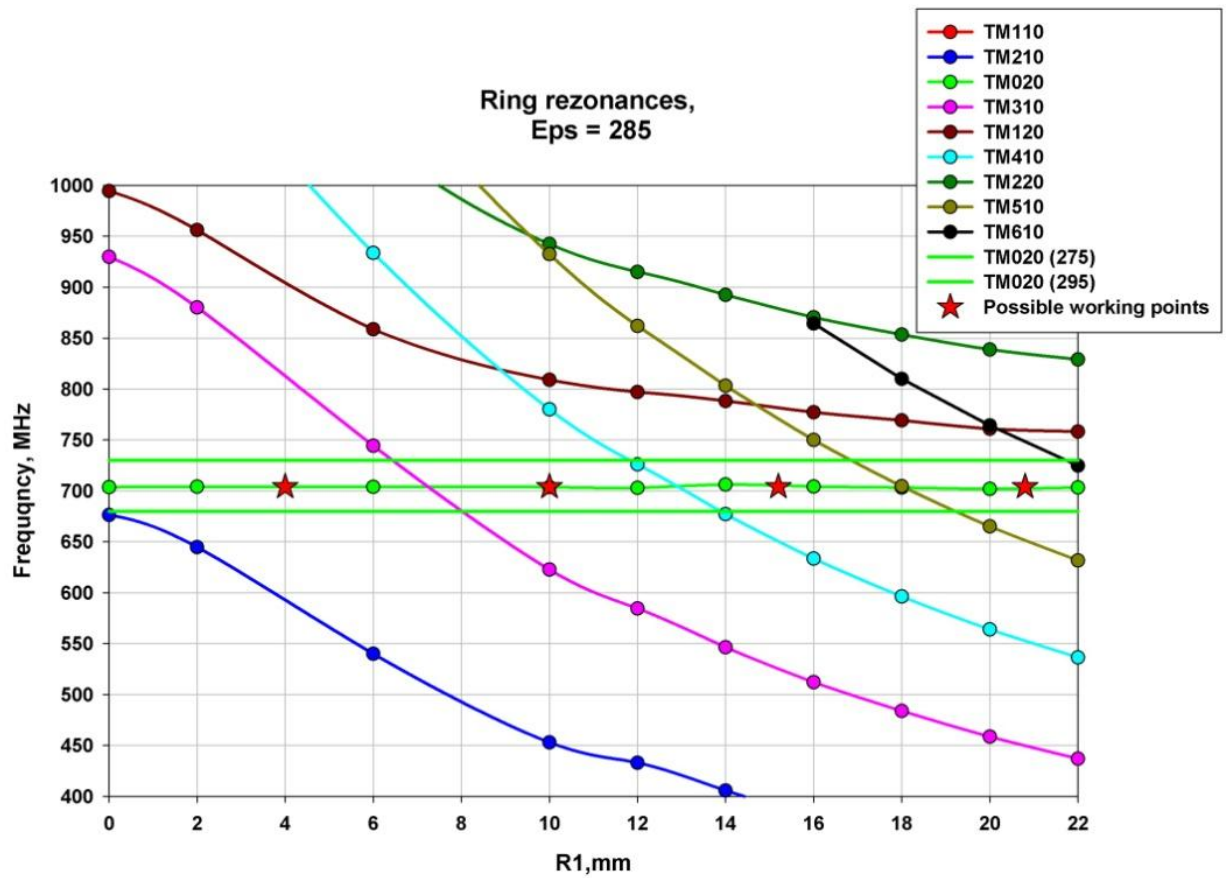

Fig. 10. Frequencies of the lowest modes in a ferroelectric ring versus the ring internal radius. 


\section{Omega-P, Inc.}

Final Report on DoE 2011 Phase I grant DE-SC0006229

Topic 44a: Nuclear Physics Accelerator Technology, Materials and Components for Radio Frequency Devices

FAST 704 MHz FERROELECTRIC TUNER FOR SUPERCONDUCTING CAVITIES

Fig. 11 shows the dependence of the external radius $R_{2}$ of the ring on the internal radius $R_{1}$ of the center hole. The frequency of operating mode $\mathrm{TM}_{020}$ is kept constant at $704 \mathrm{MHz}$.

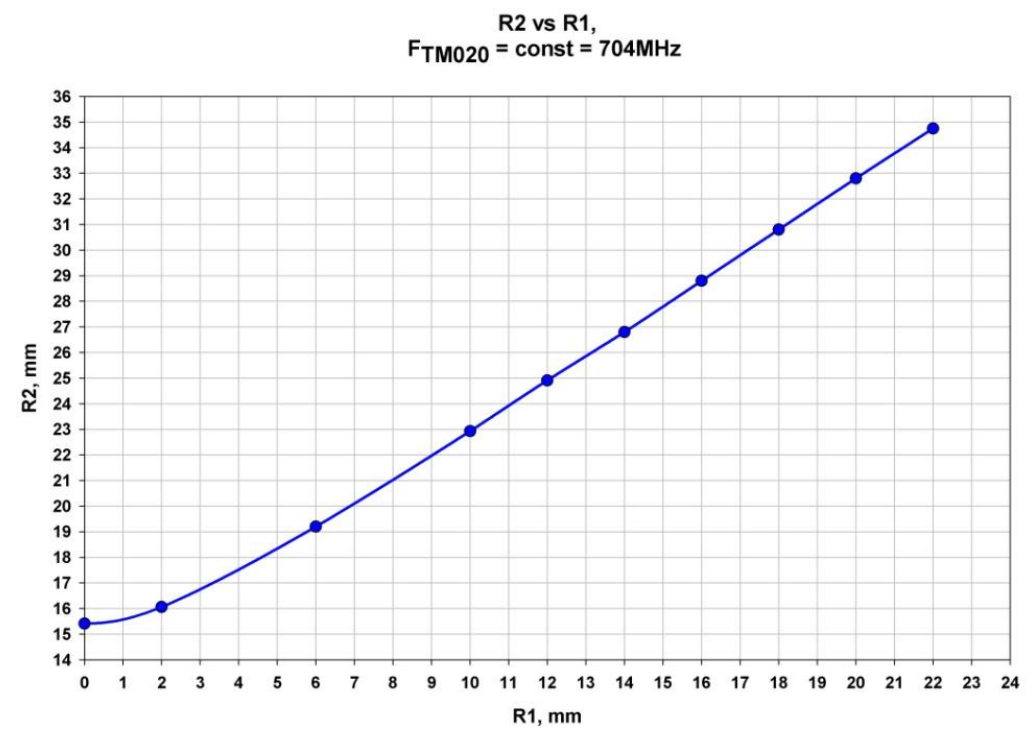

Fig. 11. Ring external radius $R_{2}$ versus the internal radius $R_{1}$ for the fixed frequency of the operating mode, namely $704 \mathrm{MHz}$. Here the dielectric constant $\varepsilon=285$.

According to these graphs, possible combinations of inner radius $R_{1}$ and outer radius $R_{2}$ are the following:

$$
\begin{array}{ll}
R_{1}=4 \mathrm{~mm}, & R_{2}=17.4 \mathrm{~mm} ; \\
R_{1}=10 \mathrm{~mm}, & R_{2}=22.8 \mathrm{~mm} ; \\
R_{1}=15.2 \mathrm{~mm}, & R_{2}=28.0 \mathrm{~mm} \\
R_{1}=20.8 \mathrm{~mm}, & R_{2}=33.7 \mathrm{~mm} .
\end{array}
$$

Among these choices, the ring with $R_{1}=10 \mathrm{~mm}$ looks preferable, because it is still small enough, and thus relatively easy to fabricate; it also can be matched to a standard 3-1/8" coaxial line. Furthermore, parasitic modes are not so close to the operating mode as for the bigger rings.

The ferroelectric assemblies, one for each phase shifter element to be combined in parallel in each tuner, comprises a ferroelectric ring brazed between two metal cups, as depicted in Fig. 12. Edges of the cups are rounded in order to have improved breakdown strength. The small rounded sitting places necessary to shield triple points near the ferroelectric edges, are not shown in the figure. 


\section{Omega-P, Inc.}

Final Report on DoE 2011 Phase I grant DE-SC0006229

Topic 44a: Nuclear Physics Accelerator Technology, Materials and Components for Radio Frequency Devices

FAST 704 MHz FERROELECTRIC TUNER FOR SUPERCONDUCTING CAVITIES
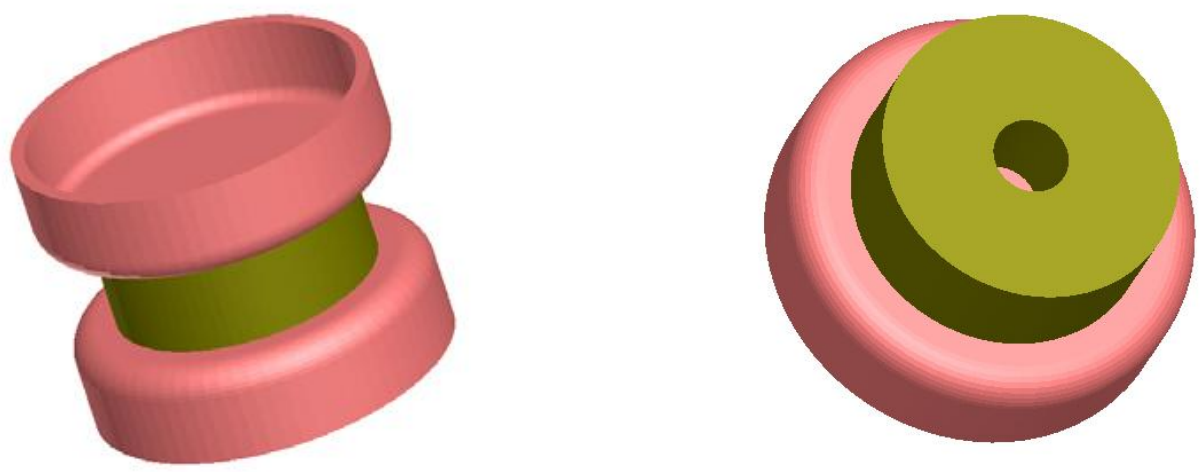

Fig.12. Concept of the ferroelectric assembly, with one required for each phase shifter module.

An example of a ferroelectric assembly positioned between two conducting plates is shown in Fig. 13. The edges of the plates near the sitting hole in the plate walls are rounded. A coaxial line terminated by the ferroelectric assembly is shown in Figure 14.

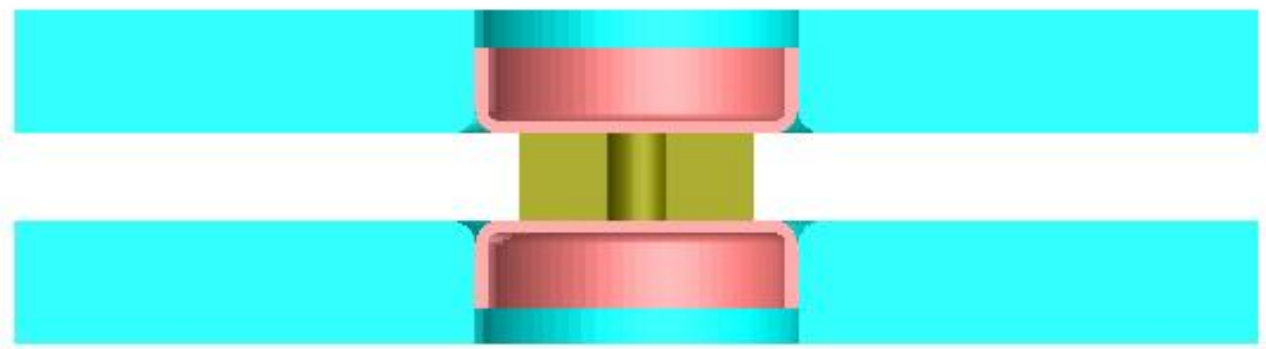

Fig. 13. A ferroelectric assembly between conducting plates. 
Phase II proposal submitted following Phase I DoE SBIR grant DE-SC0006229 in response to DoE SBIR/STTR 2011 Solicitation Topic 44a

Nuclear Physics Accelerator Technology, Materials and Components for Radio Frequency Devices

\section{FAST FERROELECTRIC L-BAND TUNER FOR SUPERCONDUCTING CAVITIES}

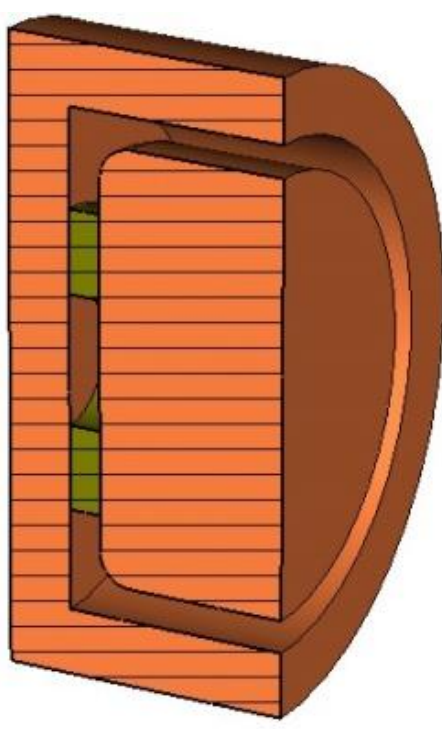

(a)

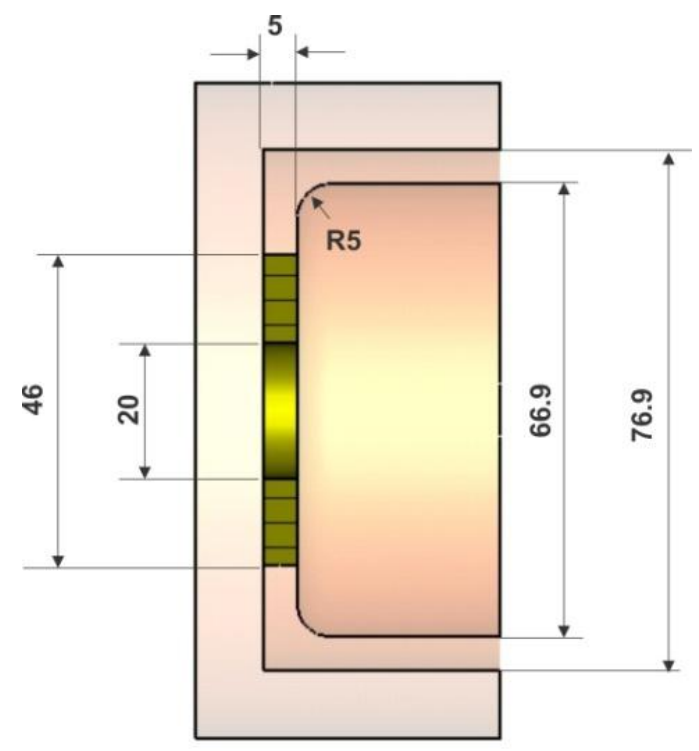

(b)

Fig. 14. (a) Schematic of a coaxial line terminated by a ferroelectric assembly. In (b) dimensions (in $\mathrm{mm}$ ) are given for the $704 \mathrm{MHz}$ example.

For an incident power level normalized to $P=1 \mathrm{~kW}$, the maximal temperature rise is $5.3^{\circ} \mathrm{C}$, and the average temperature rise over the ring is $1.9^{\circ} \mathrm{C}$. The average losses are $5.3 \%$ at the phase shift of $112^{\circ}$ (dielectric constant changes from 295 to 275). Thus, phase shift/loss are $21.2 \%$. In Fig. 15 the maximal temperature rise is shown versus the ring outer diameter. Average temperature rise versus the ring outer diameter is shown in Fig. 16. These values are for a loss tangent for the ferroelectric material of $1.5 \times 10^{-3}$.

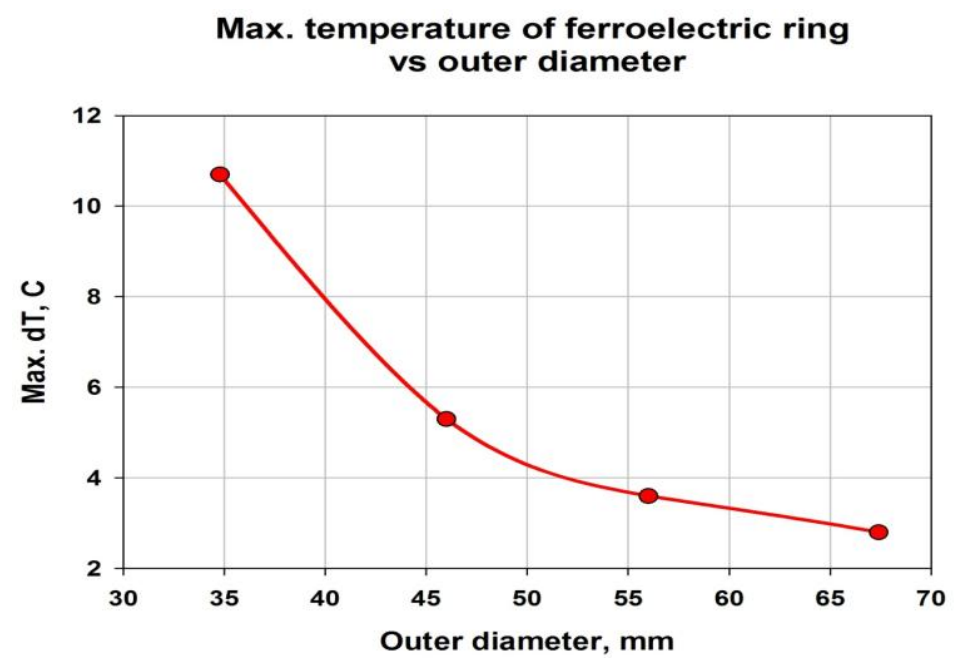

Fig. 15. Maximal temperature rise versus the ring outer diameter. 
Omega-P, Inc.

Phase II proposal submitted following Phase I DoE SBIR grant DE-SC0006229 in response to DoE SBIR/STTR 2011 Solicitation Topic 44a

Nuclear Physics Accelerator Technology, Materials and Components for Radio Frequency Devices

\section{FAST FERROELECTRIC L-BAND TUNER FOR SUPERCONDUCTING CAVITIES}

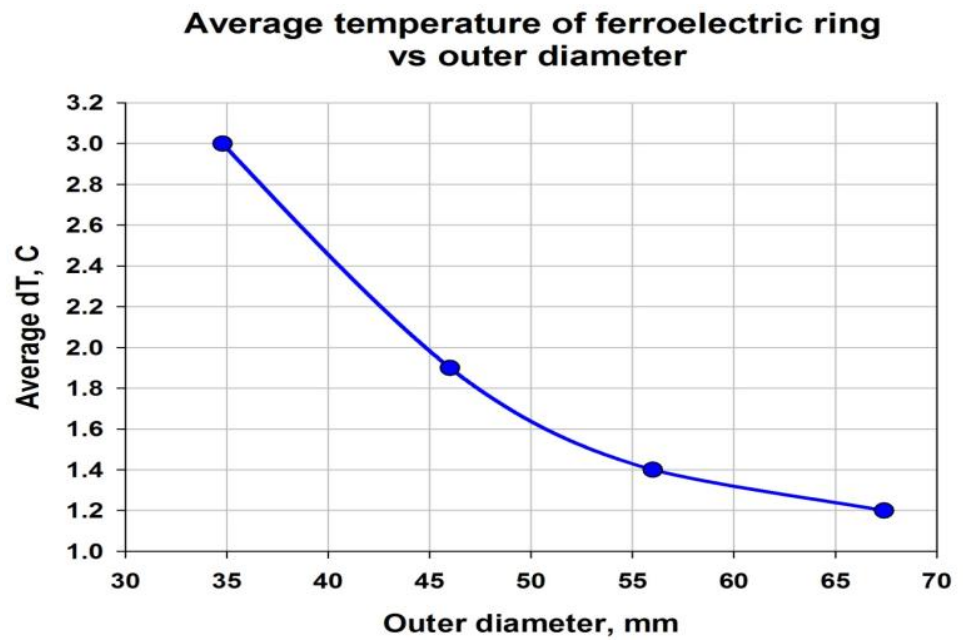

Fig. 16. Average temperature rise versus the ring outer diameter.

The temperature rise dependences versus the ring outer diameter show that the choice of the diameter of $46 \mathrm{~mm}$ is reasonable, because further increase of the diameter does not lead to significant decrease of the temperature rise.

\section{IIId. Engineering design for the coaxial phase shifter module.}

The geometry of the phase shifter module is shown in Fig. 17. Bias voltage is applied to an electrode (not shown on the left hand side) insulated from the outer coaxial electrode by a Teflon cylinder. The Teflon-filled choke prevents RF leakage. Dimensions for the phase shifter module have been worked out.

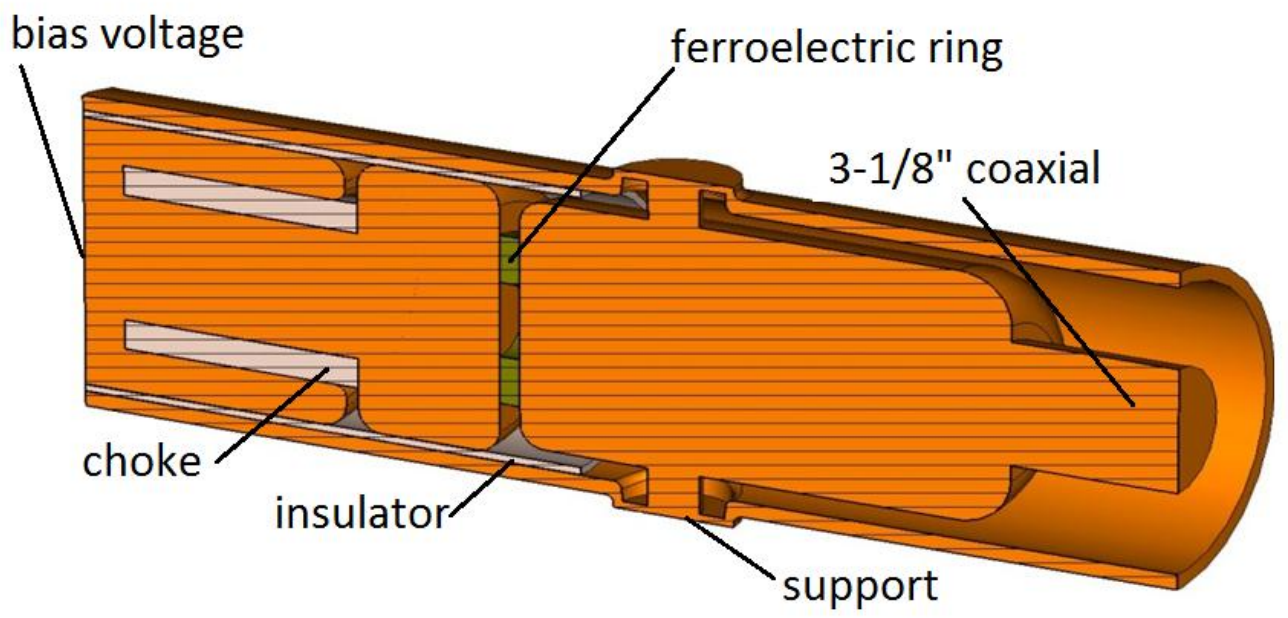

Fig. 17. Geometry of 3-1/8" coaxial phase shifter. 


\section{Omega-P, Inc.}

Phase II proposal submitted following Phase I DoE SBIR grant DE-SC0006229 in response to DoE SBIR/STTR 2011 Solicitation Topic 44a

Nuclear Physics Accelerator Technology, Materials and Components for Radio Frequency Devices

\section{FAST FERROELECTRIC L-BAND TUNER FOR SUPERCONDUCTING CAVITIES}

The losses versus frequency for the module are shown in Fig. 19 for different dielectric constants of the ferroelectric ring (i.e., for different bias). Phase shift versus frequency is shown in Fig. 20. RF leakage versus frequency is shown Fig. 21, showing that the choke works well.

704 MHz, 3-1/8" Phase Shifter

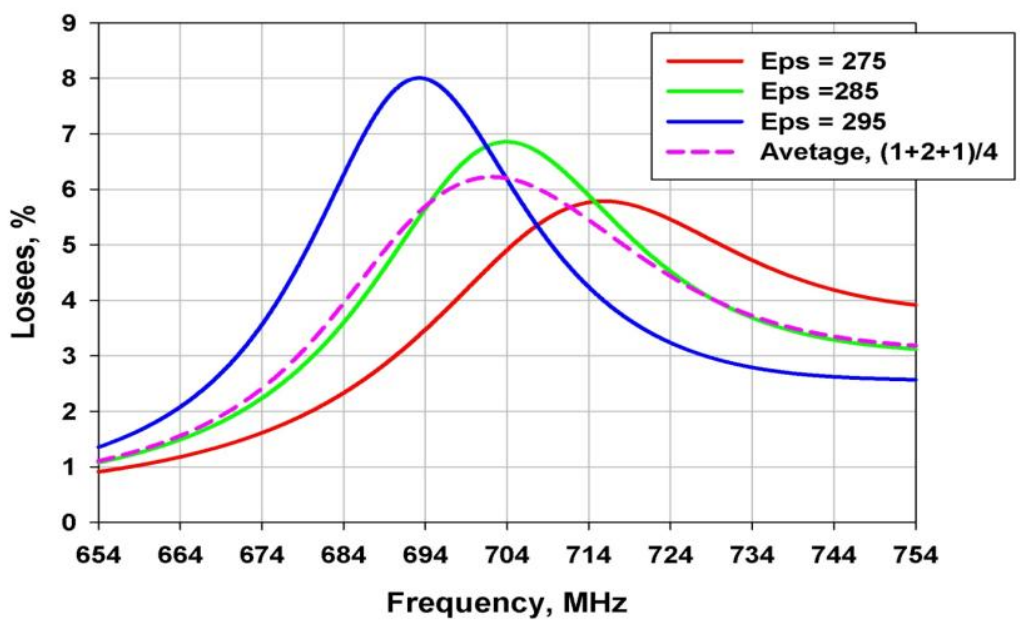

Fig. 19. Losses versus frequency for different dielectric constants of the ferroelectric ring (i.e., for different biases), for a loss tangent for the ferroelectric material of $1.5 \times 10^{-3}$.

704 MHz, 3-1/8" Phase Shifter

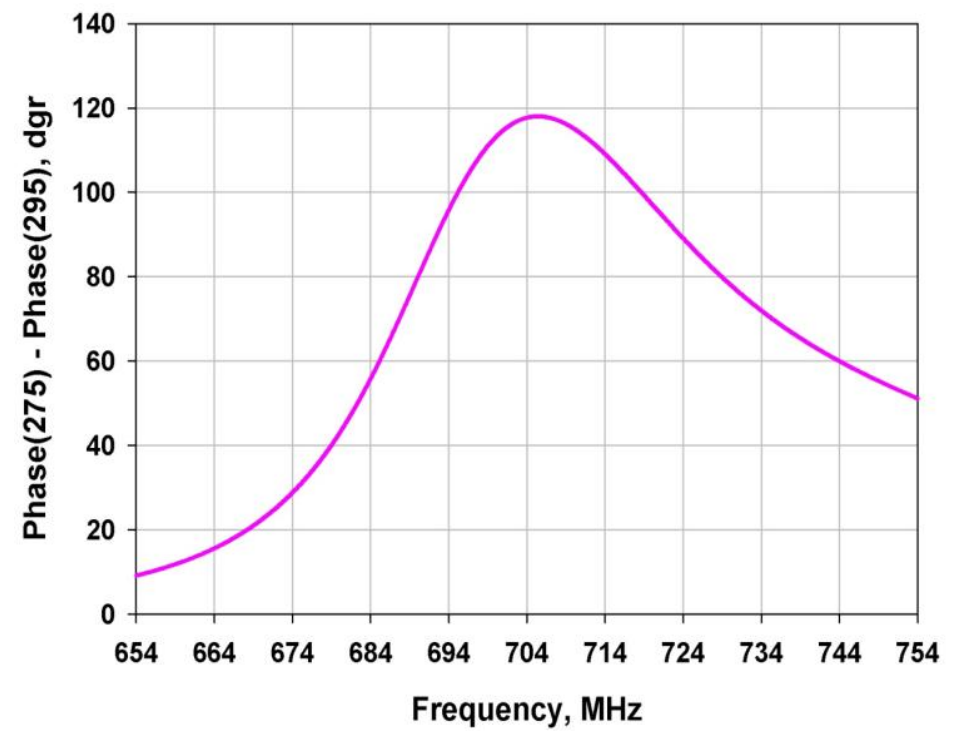

Fig. 20. Phase shift versus frequency for bias changes that switch $\varepsilon$ from 295 to 275 . . 
Omega-P, Inc.

Phase II proposal submitted following Phase I DoE SBIR grant DE-SC0006229

in response to DoE SBIR/STTR 2011 Solicitation Topic 44a

Nuclear Physics Accelerator Technology, Materials and Components for Radio Frequency Devices

FAST FERROELECTRIC L-BAND TUNER FOR SUPERCONDUCTING CAVITIES

$704 \mathrm{MHz}, 3-1 / 8$ " Phase Shifter

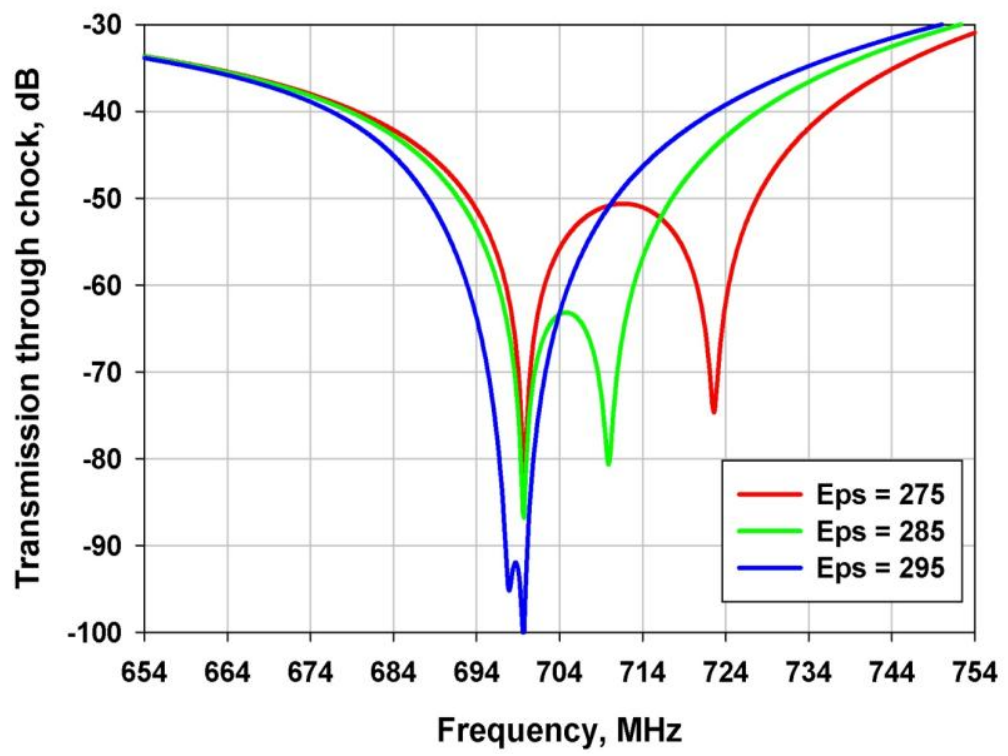

Fig 21. Transmission leakage through the slot which separates the HV part from the grounded part for different dielectric constants of ferroelectric.

For all cases transmission leakage is not more than $-50 \mathrm{~dB}$ at $704 \mathrm{MHz}$.

The RF field distribution in the phase shifter is shown in Fig 22.

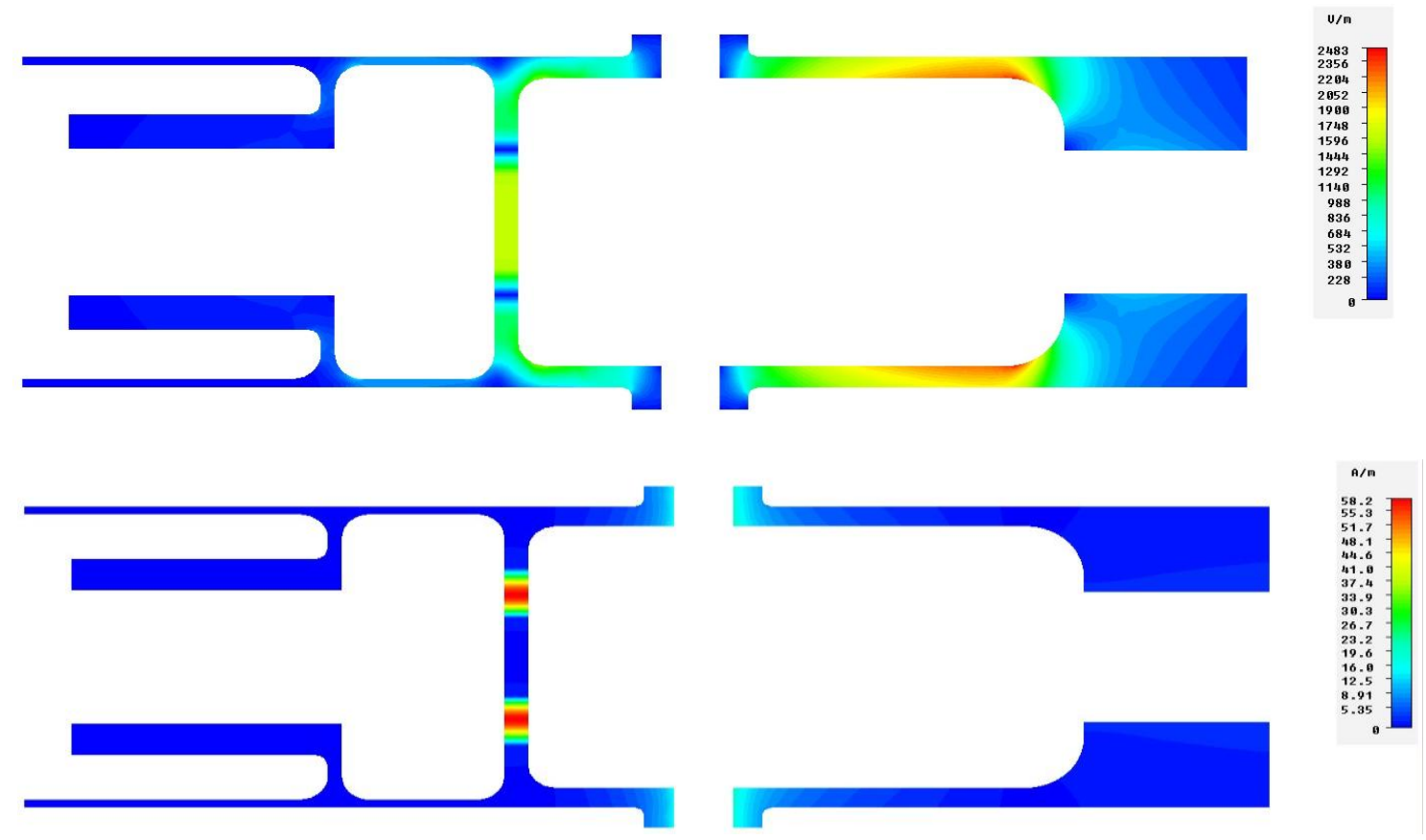

Fig 22. RF field distributions in the phase shifter module, with electric field at top and magnetic field at bottom. 
In order to change the ring dielectric constant from 295 to 275 one needs to apply a bias electric field of $18 \mathrm{kV} / \mathrm{cm}$. Thus, for the ring thickness of $0.5 \mathrm{~mm}$ the bias voltage is $9 \mathrm{kV}$. The dc bias electric field distribution in the phase shifter is shown in Fig. 23. The field near the triple point (copper + Teflon + air) is plotted in Fig. 24. One can see that the field enhancement does not exceed 1.7, which means that the field does not exceed $31 \mathrm{kV} / \mathrm{cm}$. But this field "looks" only at Teflon. Thus, hopefully high electric field in slot near Teflon will not cause breakdown, despite an E-field magnitude that is marginally above the air breakdown limit.

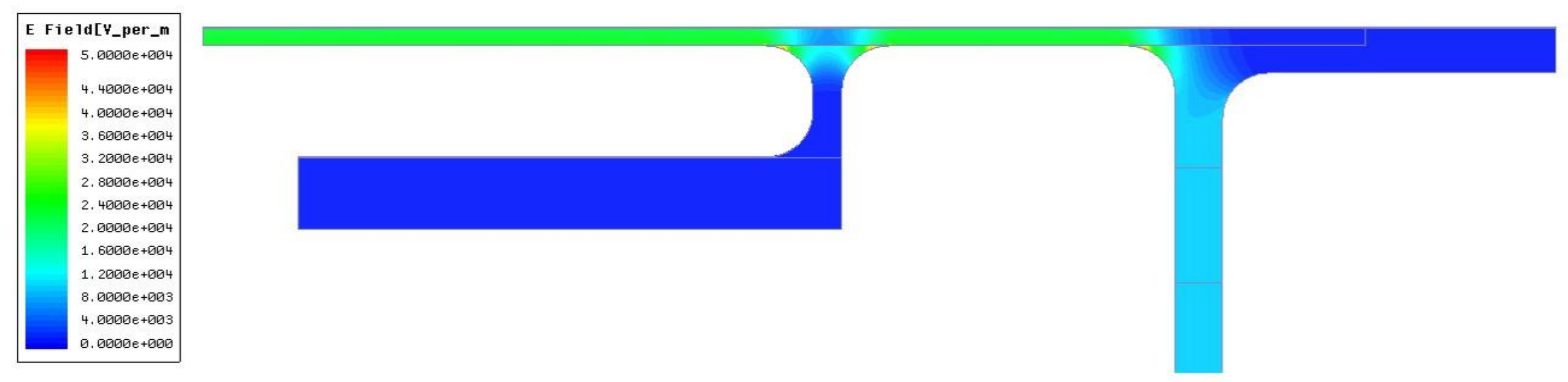

Fig. 23. Bias electric field distribution in the phase shifter for the bias voltage of $5 \mathrm{~V}$.

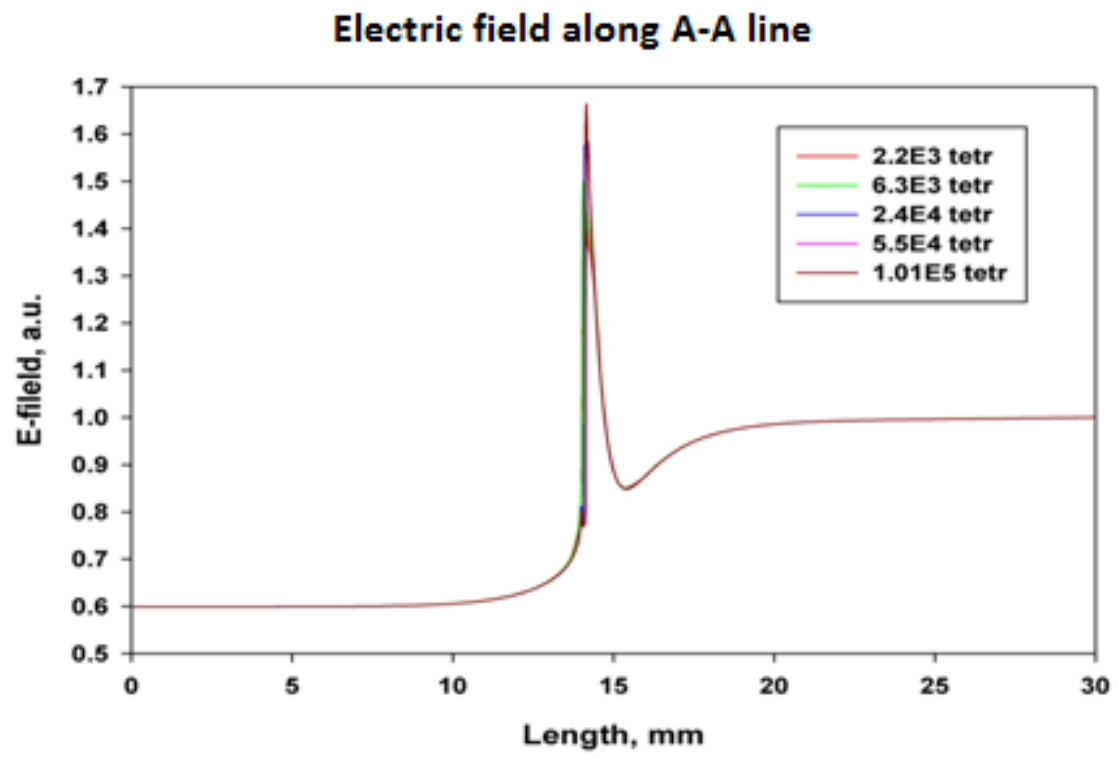

Fig. 24. Surface electric field near the triple point (see Fig. 20) for different mesh size. In the gap where the ring is placed the electric field is normalized to unity.

The cooling scheme is shown in Figure 25. The temperature distribution is shown in Fig. 26 for an input power of $1 \mathrm{~kW}$. One can see that the temperature rise does not exceed $5.2^{\circ} \mathrm{C}$. 


\section{Omega-P, Inc.}

Phase II proposal submitted following Phase I DoE SBIR grant DE-SC0006229

in response to DoE SBIR/STTR 2011 Solicitation Topic 44a

Nuclear Physics Accelerator Technology, Materials and Components for Radio Frequency Devices

\section{FAST FERROELECTRIC L-BAND TUNER FOR SUPERCONDUCTING CAVITIES}

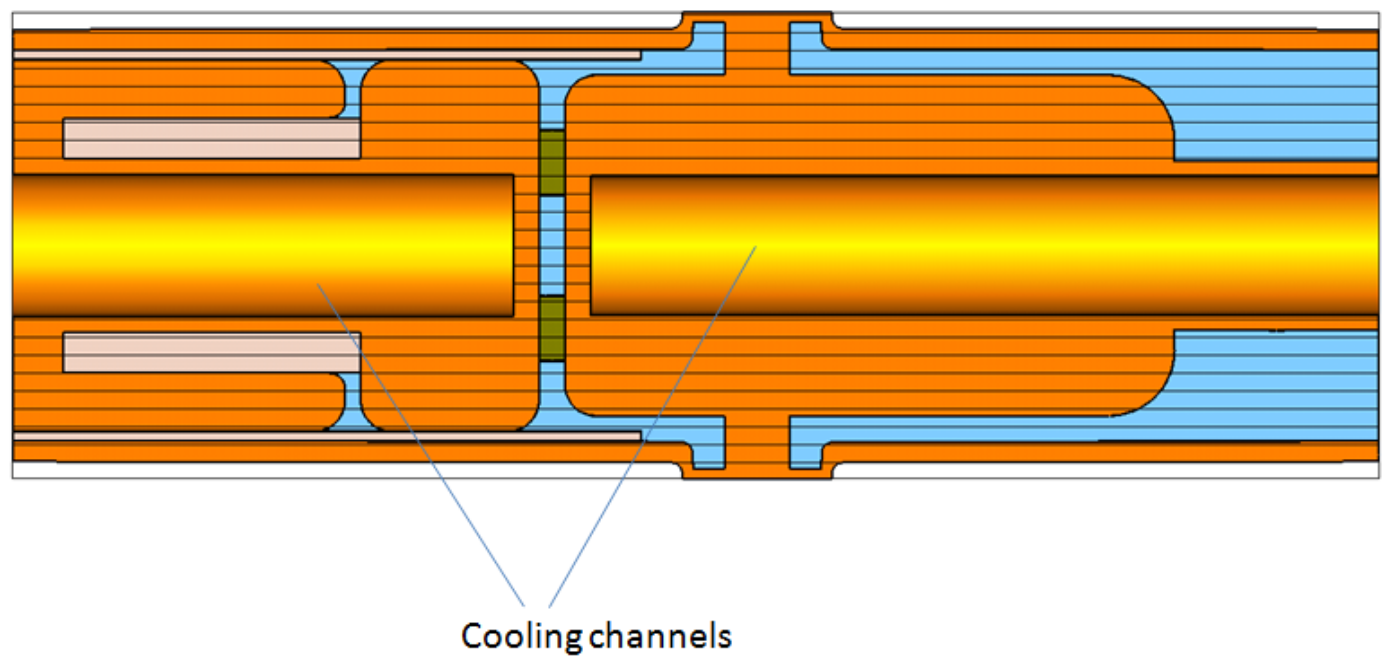

Fig. 25. Cooling scheme. Orange - copper, gray - ferroelectric, pink - Teflon,

blue - air. It is assumed that the cooling channels have constant temperature at their surfaces. Thermal conductivity of ferroelectric is $7.2 \mathrm{~W} /\left(\mathrm{K}^{*} \mathrm{~m}\right)$.
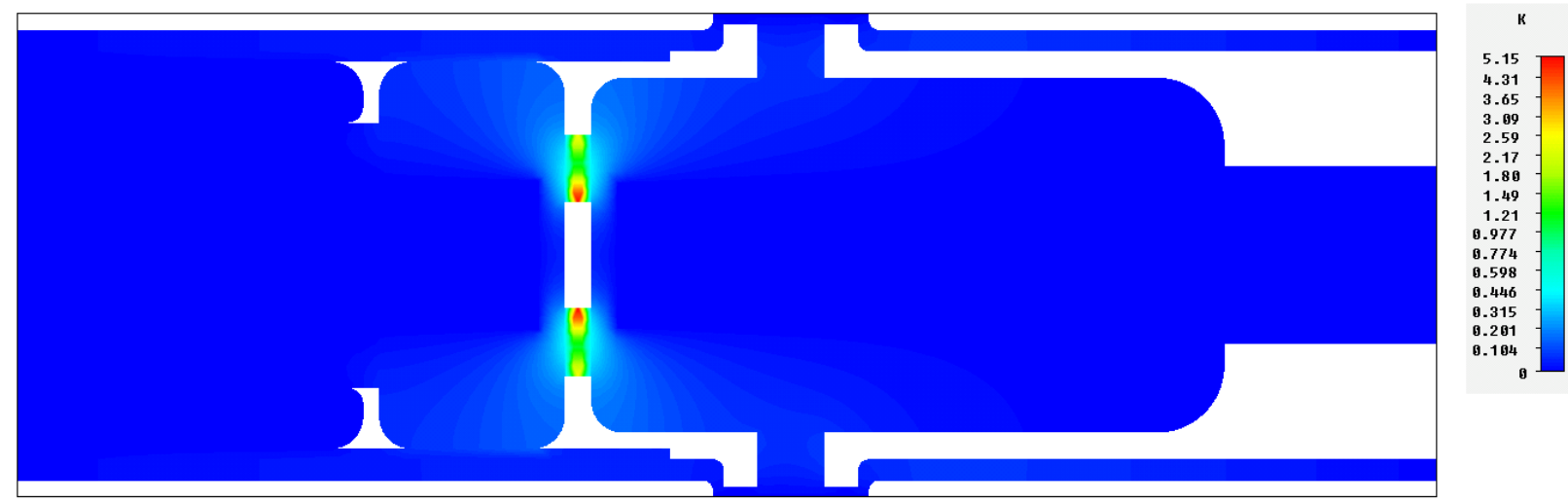

Fig. 26. Temperature distribution for an input power of $1 \mathrm{~kW}$.

A conceptual mechanical design of the phase shifter module is shown in Fig. 27. The full tuner assembly is shown in Fig. 28, with a total of ten phase shifters in this example, to enable control at the cavity input of a power level as high as $50 \mathrm{~kW}$. 


\section{Omega-P, Inc.}

Phase II proposal submitted following Phase I DoE SBIR grant DE-SC0006229 in response to DoE SBIR/STTR 2011 Solicitation Topic 44a

Nuclear Physics Accelerator Technology, Materials and Components for Radio Frequency Devices

\section{FAST FERROELECTRIC L-BAND TUNER FOR SUPERCONDUCTING CAVITIES}

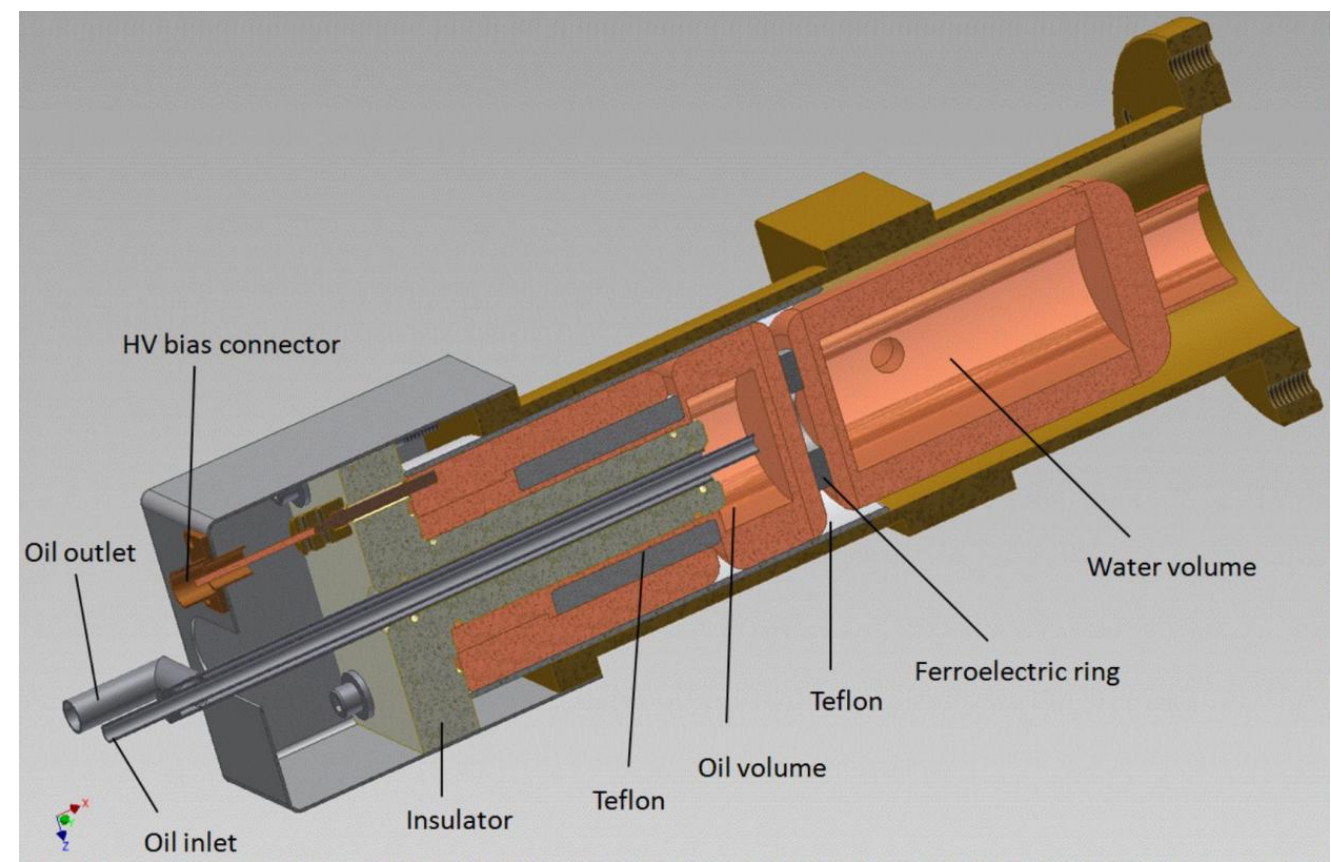

Fig. 27. A 3D model of the phase shifter module. Water inlet and HV socket are shown.

In order to operate one module at a power level of $5 \mathrm{~kW}$, the computations shown here indicate that a temperature rise of $30^{\circ} \mathrm{C}$ will need to be tolerated But, because of the temperature dependence of the dielectric constant of the ferroelectric $\left(1.5 /{ }^{\circ} \mathrm{C}\right)$, it may be that a pre-heated coolant will be used to avoid serious changes in the dielectric constant when the RF power is on. These computations also show that, in order to control $50 \mathrm{~kW}$ of incident power for each cavity, one would employ five $5-\mathrm{kW}$ phase shifters in each port of a magic-T, as shown in Fig. 28.

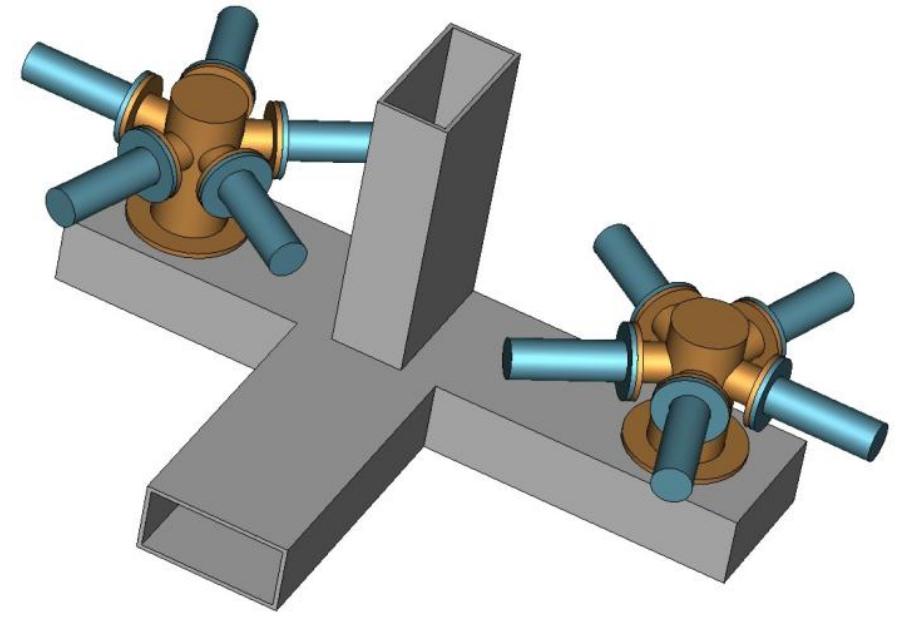

Fig. 28. Magic-T with 5 phase shifters in each port, so as to sustain an input power of $50 \mathrm{~kW}$. 


\section{Omega-P, Inc.}

Phase II proposal submitted following Phase I DoE SBIR grant DE-SC0006229 in response to DoE SBIR/STTR 2011 Solicitation Topic 44a

Nuclear Physics Accelerator Technology, Materials and Components for Radio Frequency Devices

\section{FAST FERROELECTRIC L-BAND TUNER FOR SUPERCONDUCTING CAVITIES}

The five-way power combiner design for this application is shown in Fig. 29, with its reflection coefficient versus the frequency shown in Fig. 30. At $704 \mathrm{MHz}$, input reflections are seen to be down to below $-45 \mathrm{~dB}$.

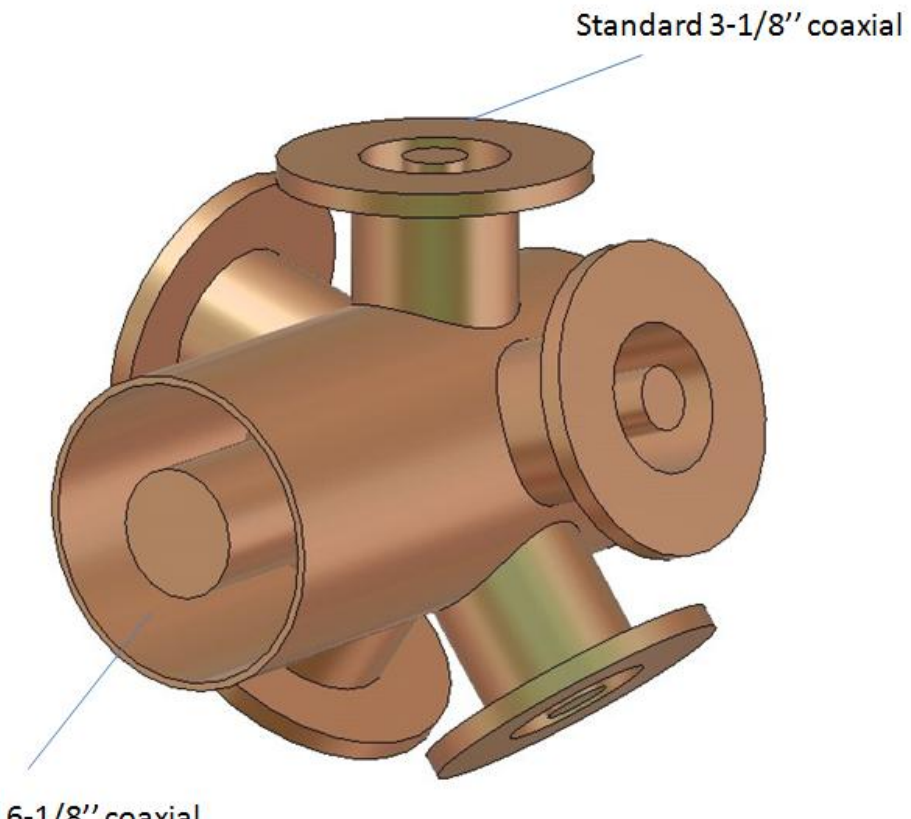

Standard 6-1/8" coaxial

Fig 29. The power combiner design.

S11,

Combiner 5-to-1

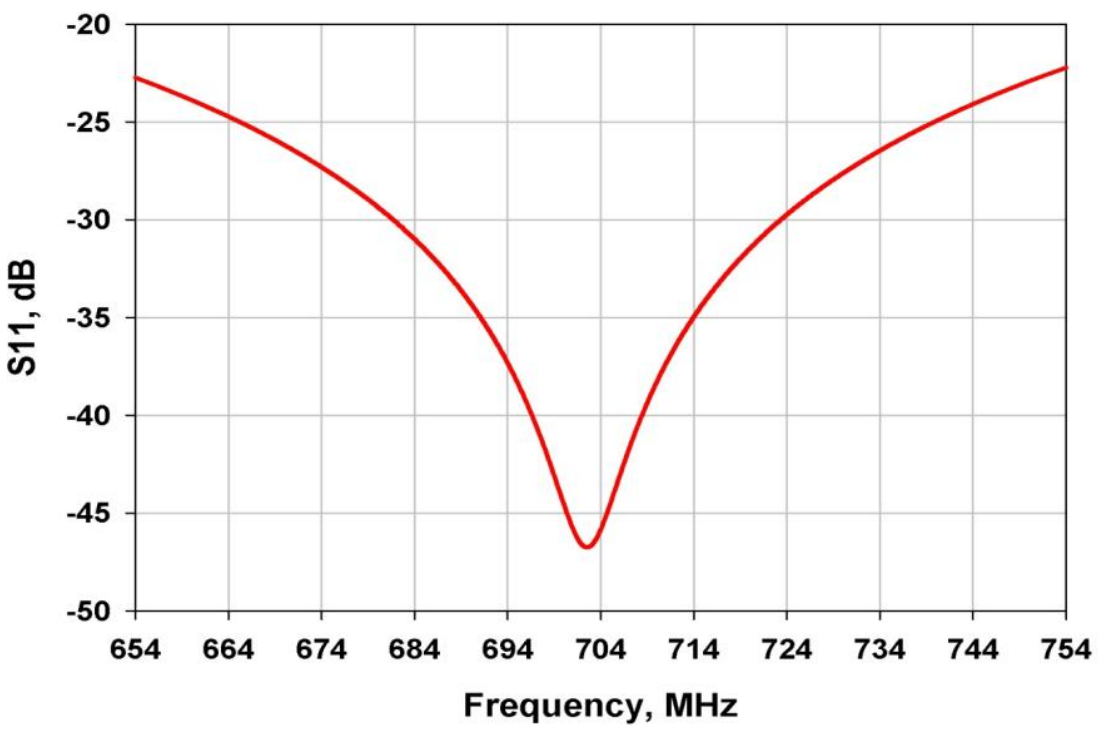

Fig. 30. Reflection coefficient versus frequency for the five-way power combiner. 


\section{Omega-P, Inc.}

Phase II proposal submitted following Phase I DoE SBIR grant DE-SC0006229 in response to DoE SBIR/STTR 2011 Solicitation Topic 44a

Nuclear Physics Accelerator Technology, Materials and Components for Radio Frequency Devices

\section{FAST FERROELECTRIC L-BAND TUNER FOR SUPERCONDUCTING CAVITIES}

\section{IIIe. Measurements of the dielectric constant and loss tangent of metal-coated ferroelectric samples}

Measurements without bias. A variety of ferroelectric samples were measured to determine their dielectric constant and loss tangent. All samples were shaped as bars with rectangular cross-sections. Fig. 31a shows the bar configuration, with photos in Fig. 31b and 31c. Table I lists the sizes of the bars upon which measurements were made.

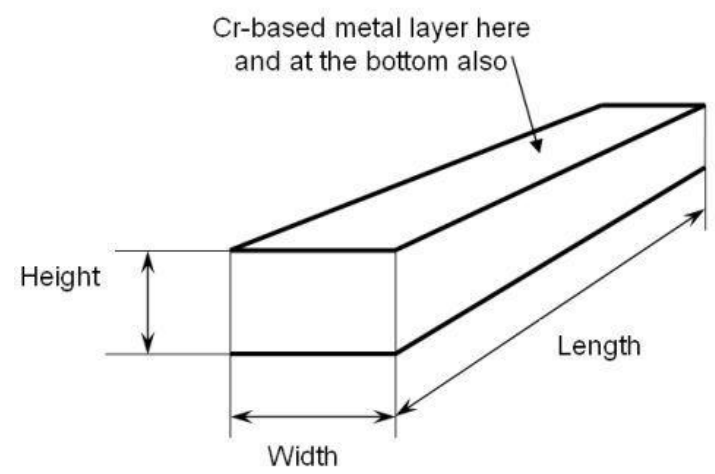

a)

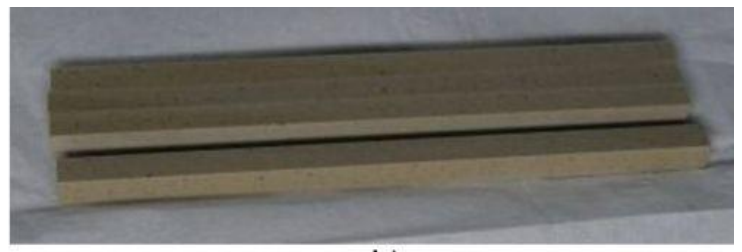

b)

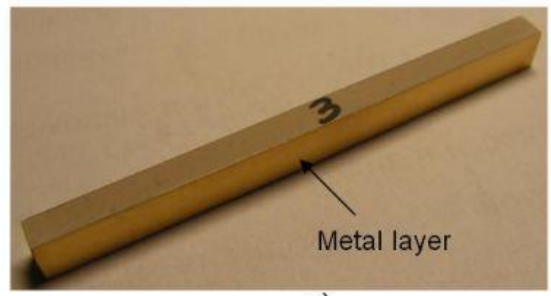

c)

Fig 31. Bar dimensions in (a); photos of non-coated bars in (b) and of a bar with metalized coatings in (c).

Table I. Sizes of available bars.

\begin{tabular}{|c|c|c|c|c|}
\hline $\begin{array}{c}\text { Sample } \\
\text { code name }\end{array}$ & Height, $\mathrm{mm}$ & Width, $\mathrm{mm}$ & Length, mm & Quantity \\
\hline T-IV-1 & 6.0 & 5.1 & 108.0 & 1 \\
\hline T-I-1 & 6.0 & 5.1 & 108.1 & 1 \\
\hline $1^{\text {st }}$ set & 5.7 & 5.1 & 108.0 & 4 \\
\hline $2^{\text {nd }}$ set & 5.7 & 5.1 & 108.0 & 4 \\
\hline short & 3.8 & 6.0 & 66.5 & 6 \\
\hline
\end{tabular}

Two opposing long sides of each bar were coated with a metal film. The metal film has three layers: the first one is a layer of $\mathrm{Cr}$ (chrome), the second one deposited on the top of the first is a layer of $\mathrm{Cu}$ (copper), and the last layer is a layer of $\mathrm{Au}$ (gold). The deposition of $\mathrm{Cr}$ and $\mathrm{Cu}$ was done in a magnetron-driven plasma chamber shown in Fig. 32, while the deposition of gold was done by electroplating (i.e., deposition from an electrolyte). 
Phase II proposal submitted following Phase I DoE SBIR grant DE-SC0006229 in response to DoE SBIR/STTR 2011 Solicitation Topic 44a

Nuclear Physics Accelerator Technology, Materials and Components for Radio Frequency Devices

FAST FERROELECTRIC L-BAND TUNER FOR SUPERCONDUCTING CAVITIES

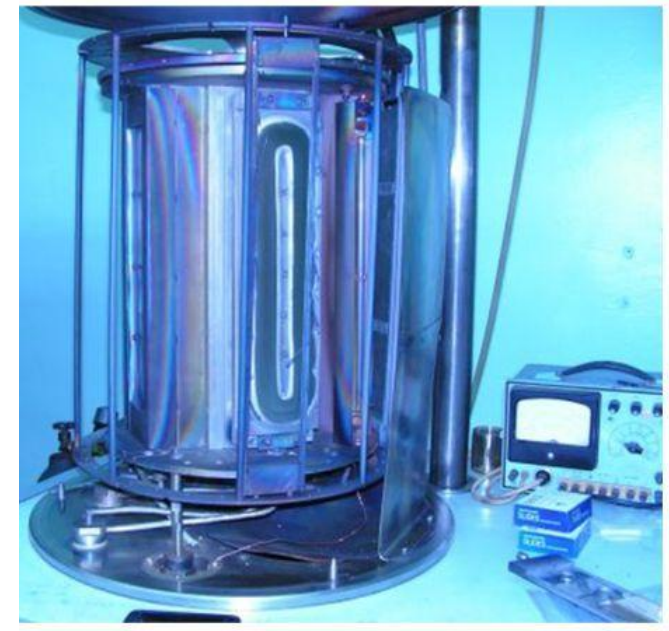

a) b)
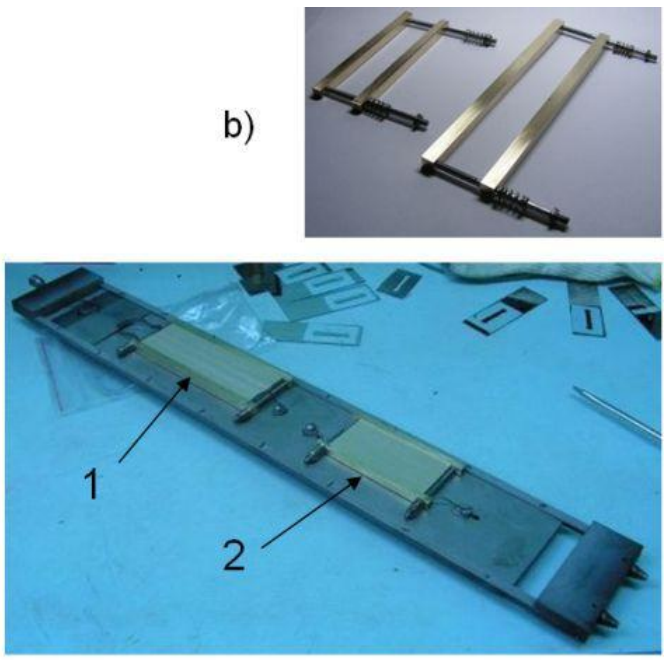

c)

Fig. 32 (a) magnetron chamber to deposit $\mathrm{Cr}$ and $\mathrm{Cu}$; (b) frames to hold bars during the deposition process; (c) the bars inserted in the frames form cartridges (e.g. 1 and 2), where each bar has only two sides exposed to the metalizing process.

To measure the dielectric constant and loss tangent, the bars were placed in rectangular cavities shown in Fig. 33, with a known mode spectrum. Each cavity was equipped with two loops on each side of the ceramic bar to measure S-parameters of known modes. The data were compared with HFSS simulations (Fig. 34), and by performing parameter scans to match the peaks and the width of resonance curves in simulations and measurements, the dielectric constant and loss tangent at the mode frequencies were inferred for every sample. Results are shown in Figs. 35 and 36.

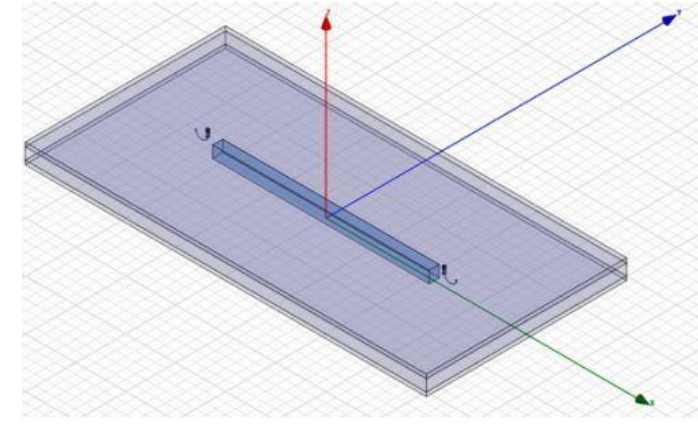

a)

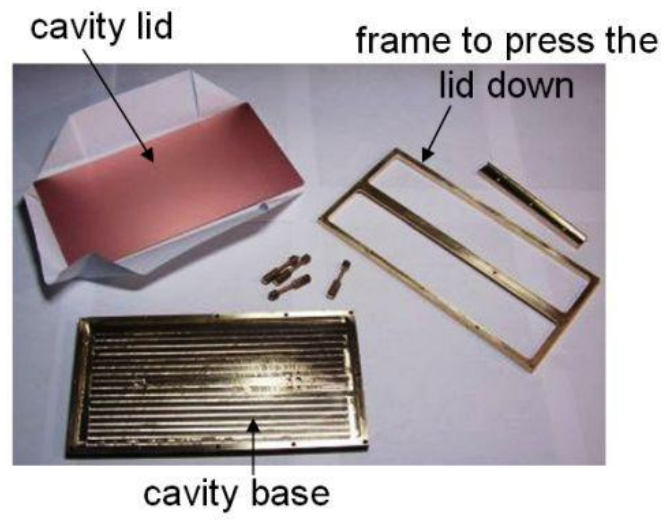

b)

Fig. 33. (a) HFSS model of a cavity equipped with two loops (connected to $50 \mathrm{Ohm}$ cables) on each side of a ceramic bar to measure S-parameters of known modes and infer later the relevant parameters of metal-coated ceramic bar. (b) photos of the actual parts of one of the cavities used in measurements. 


\section{Omega-P, Inc.}

Phase II proposal submitted following Phase I DoE SBIR grant DE-SC0006229 in response to DoE SBIR/STTR 2011 Solicitation Topic 44a

Nuclear Physics Accelerator Technology, Materials and Components for Radio Frequency Devices

\section{FAST FERROELECTRIC L-BAND TUNER FOR SUPERCONDUCTING CAVITIES}

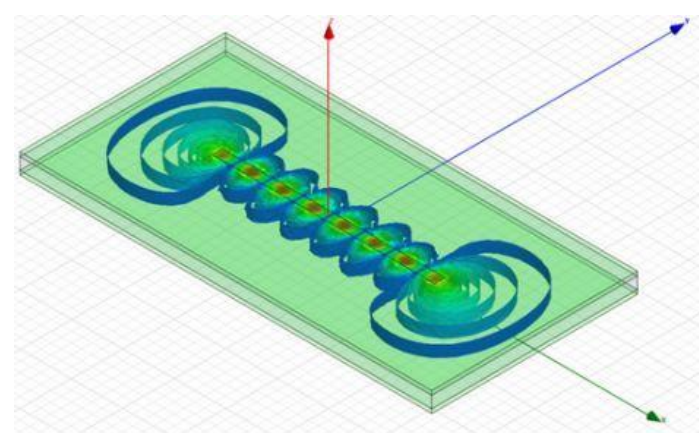

a)

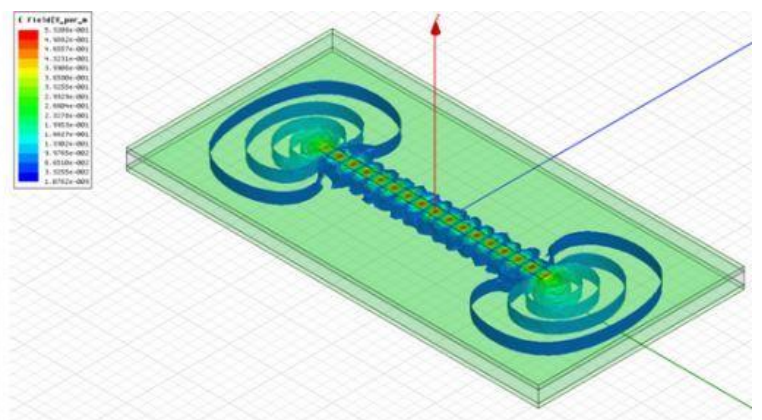

b)

Fig. 34. The electric field pattern of some of the modes (as simulated by HFSS) in one of the cavities used in measurements

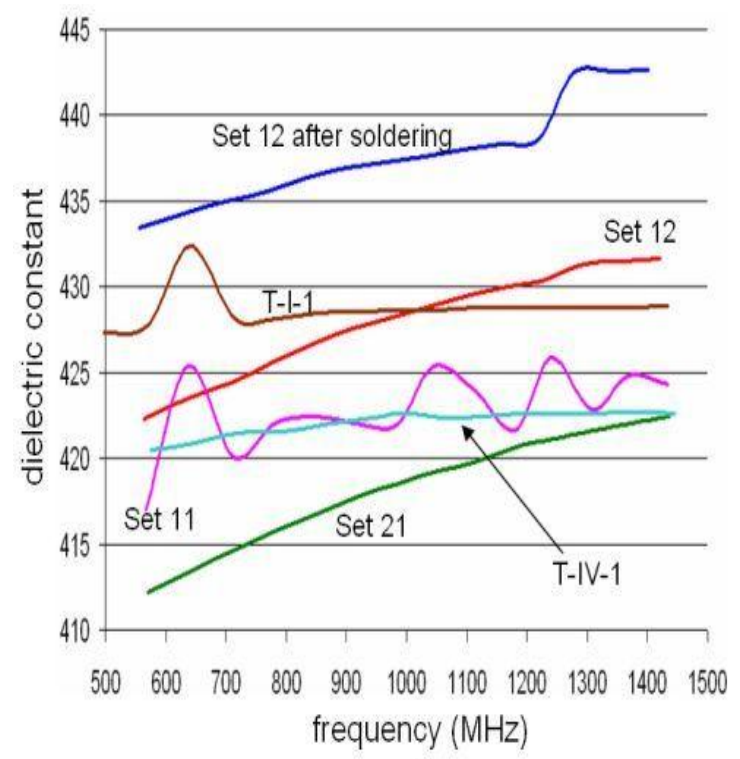

a)

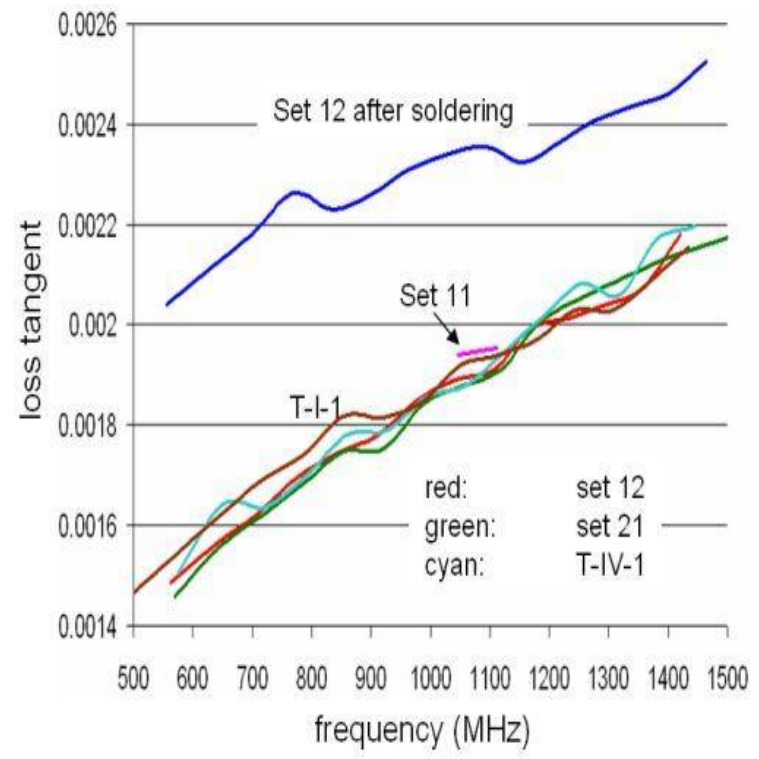

b)

Fig. 35. The dielectric constant and loss tangent at the mode frequencies as inferred for each long sample. 


\section{FAST FERROELECTRIC L-BAND TUNER FOR SUPERCONDUCTING CAVITIES}

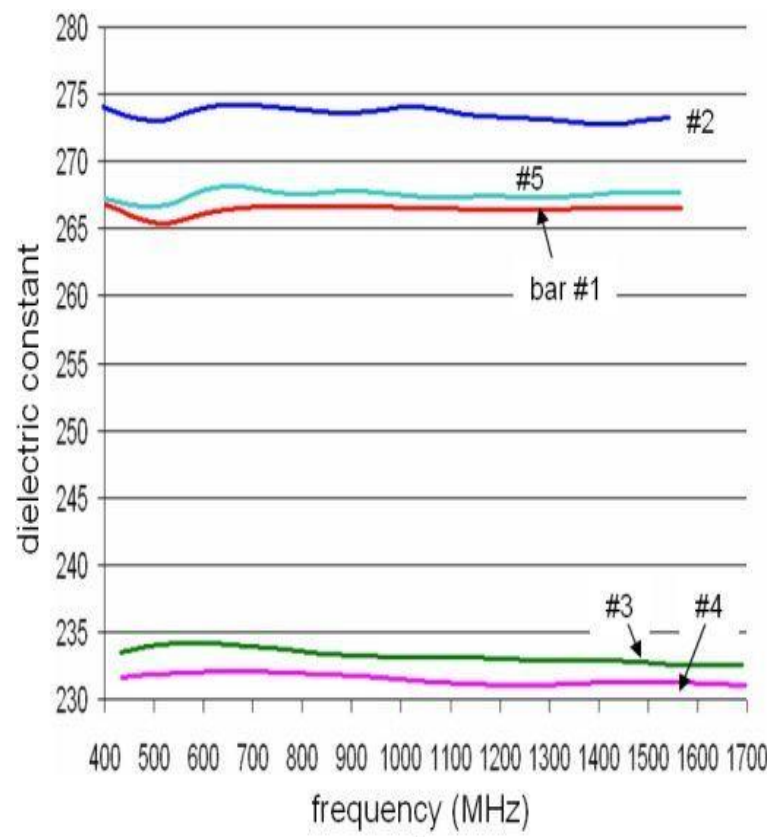

a)

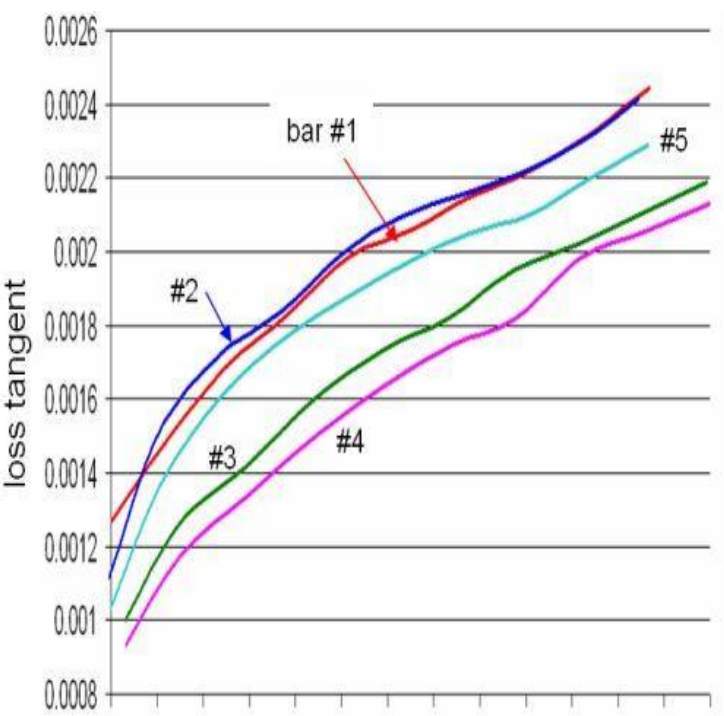

40050060070080090010001100120013001400150016001700 frequency $(\mathrm{MHz})$

b)

Fig. 36. The dielectric constant and loss tangent at the mode frequencies as inferred for short samples

Measurements under dc bias: technique. These measurements are a continuation of our efforts to characterize and confirm the suitability of the behavior of the dielectric constant and loss tangent when an external bias is applied to a bulk metal-coated ferroelectric sample, as supplied to Omega-P by Euclid TechLabs.

Prior to describing the measurements done in this recent cycle of our activities, it should be emphasized that already with these samples and similar coating, a fast response of the ferroelectric ceramic was demonstrated. Most notable data are cited by us in [14] and [15]. In the cited references the speed of response was measured as follows. CW-power from an RF generator was split in two. One portion was directed through a commercial mechanicallyadjustable phase shifter and attenuator to a mixer, while the second portion was fed to a structure that had ferroelectric elements of interest installed. After this second portion passed through the structure, it was picked up from the output port, and then also directed to the mixer. The resulting signal from the mixer was detected by a diode and monitored on an oscilloscope, and also captured by a computer for further signal processing (mainly FFT). The changes in the mixer resulting signal were monitored as a fast-changing bias was applied, and allowed to infer the response speed of the metalized ceramic.

Fig. 37 shows an example of the response to a $\sim 10 \mathrm{kV}$ fast bias pulse (scaled vertically by a factor 0.005 in the cited figure [black curve]) with fall/rise time in a range of $100 \mathrm{~ns}$. The difference signal of $67 \mathrm{mV}$ from the mixer (see the peak of the blue curve) corresponded to a phase change of $77^{\circ}$. From these data, where the response time of the phase shifter was 
Omega-P, Inc.

Phase II proposal submitted following Phase I DoE SBIR grant DE-SC0006229

in response to DoE SBIR/STTR 2011 Solicitation Topic 44a

Nuclear Physics Accelerator Technology, Materials and Components for Radio Frequency Devices

FAST FERROELECTRIC L-BAND TUNER FOR SUPERCONDUCTING CAVITIES

dominated by the $90 \mathrm{~ns}$ rise time of the voltage pulse, one can infer that the response time to a step function voltage would be equal to or less than the delay time, namely approximately $30 \mathrm{~ns}$. This could be interpreted to correspond to an average switching rate of less than $0.5 \mathrm{~ns}$ for each degree of RF phase.

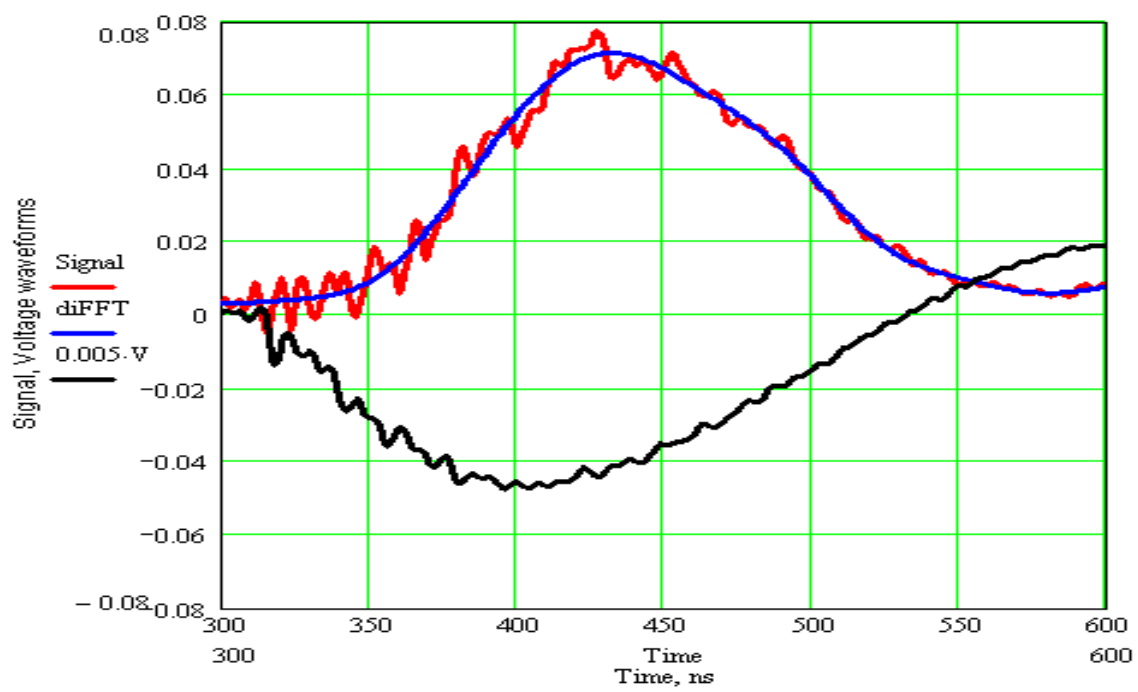

Fig. 37. Time-response of a device equipped with metalized ferroelectric ceramic identical to the ceramic used to manufacture the long bars investigated in the course of our recent activities. Red curve (convex) is the difference between data with RF off and RF on. Blue curve is FFT/IFFT processed signal. Black curve (concave) is the high-voltage pulse (scaled in this plot by 0.005) with its peak value of $\sim 9.7 \mathrm{kV}$.

Having established a fact that the ferroelectric metal-coated ceramic has a fast response speed, the recent measurements were directed to investigate the behavior of the ferroelectric samples on longer timescales. Using data presented in Figs. 35 and 36, the samples that have the closest parameters were paired. Each pair was subjected to bias (constant or changing) in configurations shown in Fig. 38-41.

Pairs of samples were tested where the samples were both soldered together or not. In the first case each bar was pre-tinned with an In-Tn alloy; the samples were then pressed against a strip of the metal (that later served as the middle electrode to which high voltage bias was applied) and subjected to heat to solder the two parts together. In the second case, the samples were placed inside the chamber, one at a time, with a strip of metal between them to serve as the middle electrode to apply bias later. In either case, the middle electrode has a tongue (see position \#4 in Figs. 39 b and 41b) to attach a bias wire. The bias wire was the middle wire of a cable, whose outer shell served as protection against arcing. In addition, the cable outer shell on the side attached to the tongue was trimmed so to form an RF-filter (see e.g. position \#5 in Fig 39 ) at the frequencies of interest $(300-1800 \mathrm{MHz})$; as the result the coupling between the loops and the bias wire was less than $-25 \mathrm{~dB}$ which insured negligible losses to an external bias circuit. Having low losses to the external circuitry was important to enable measurement of the loss tangent accurately when the bias was applied. The middle electrode had a smaller footprint than 


\section{Omega-P, Inc.}

Phase II proposal submitted following Phase I DoE SBIR grant DE-SC0006229 in response to DoE SBIR/STTR 2011 Solicitation Topic 44a

Nuclear Physics Accelerator Technology, Materials and Components for Radio Frequency Devices

\section{FAST FERROELECTRIC L-BAND TUNER FOR SUPERCONDUCTING CAVITIES}

the bars to mask the edges of electrode by the ceramic thereby helping to avoid arcing. To insure good contact between the cavity walls and the bars' metalized surfaces, strips of RF-fingers were used. $\mathrm{T}$ he cavity inside was electroplated with gold to avoid surface oxidation, and have the same wall conductivity throughout the whole cycle of measurements.

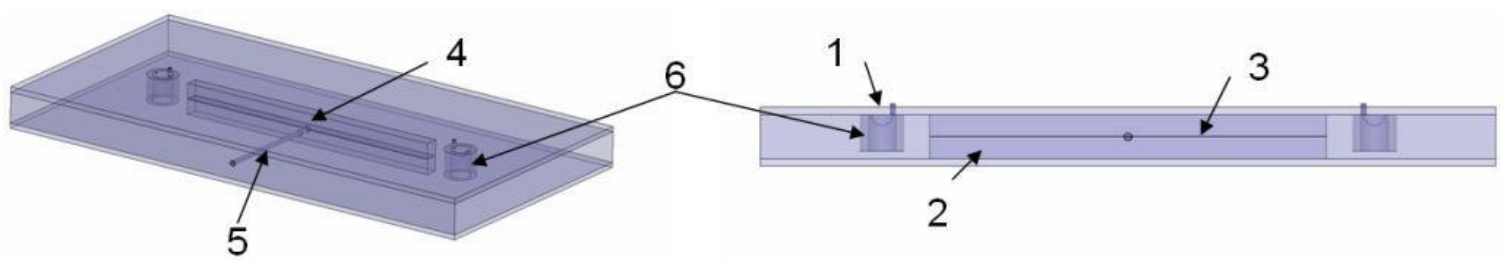

a)

b)

Fig. 38. Configuration used to test long (tall) samples: 1 - excitation loop; 2 - long ferroelectric bars (coated with Cr-based metallization), 3 - a middle electrode, 4 - a tongue on the middle electrode to attach (via soldering) a wire that delivers bias; 5 - cable whose middle wire was used to deliver bias; 6 - protective Teflon caps around loops to avoid high-voltage arcing to a network analyzer.

More details are depicted in the photos of Fig. 39.

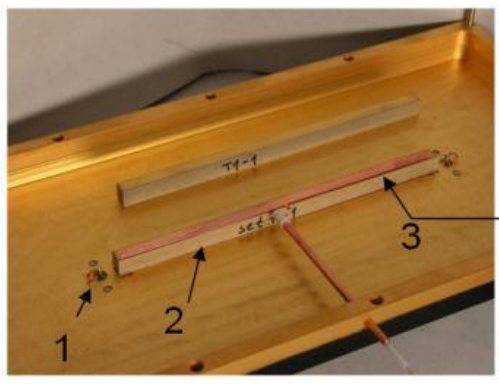

a)

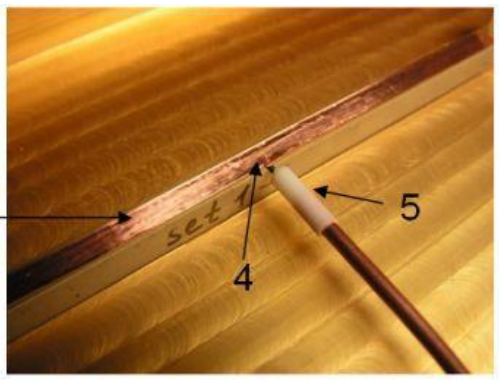

b)

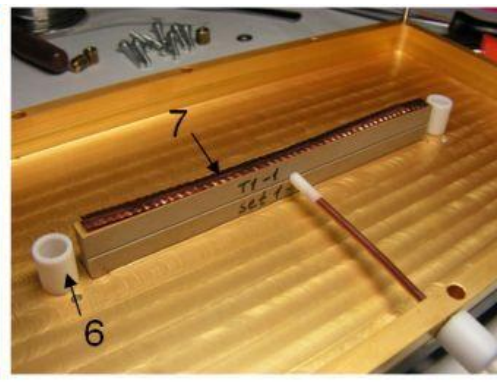

c)

Fig. 39. Configuration used to test long (and tall) samples: 1- excitation loop; 2 - ferroelectric sample; 3- middle electrode [the sizes of the electrode are such that its has a smaller footprint than the ceramic samples to mask the edges of electrode by the ceramic thereby helping to avoid arcing]; 4 - tongue to solder the bias wire; 5- cable whose middle wire is used to deliver bias,

and whose outer shell is trimmed so to achieve an effect of RF -filter at the frequencies of interest $(300-1800 \mathrm{MHz})$; as the result the coupling between the loops and the bias wire was less than $-25 \mathrm{~dB} ; 6$ - protective Teflon caps; 7 - strip of RF-fingers. 


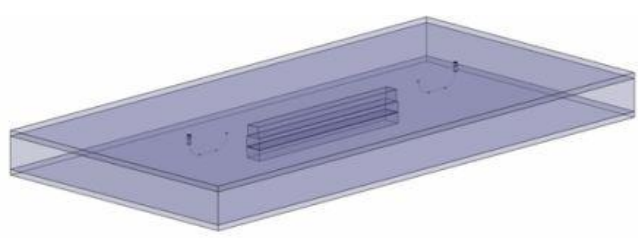

a)

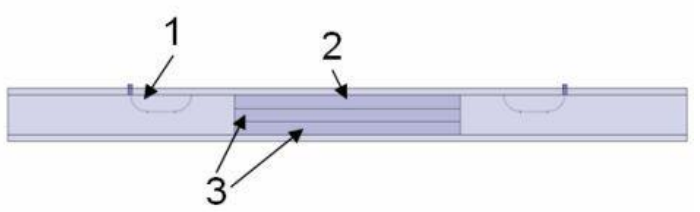

b)

Fig. 40. Configuration used to test short samples: 1- excitation loop; 2- metal insert to accommodate for difference in height; 3 - ferroelectric samples.

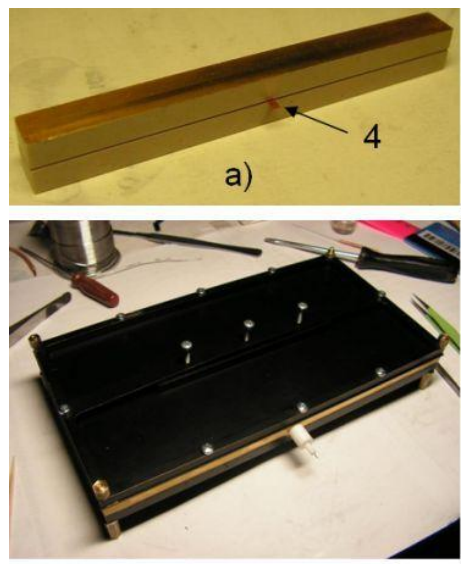

b)

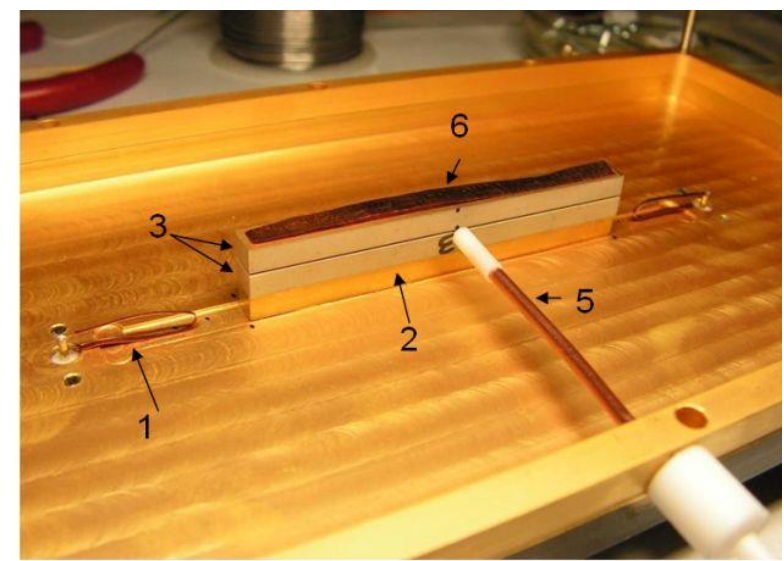

c)

Fig. 41. Configuration used to test short samples: 1- excitation loop; 2- metal insert to accommodate for difference in height; 3 - ferroelectric samples; 4 - a tongue on the middle electrode to attach (via soldering) a wire that delivers bias; 5 - cable whose middle wire was used to deliver bias; 6 - strip of RF-fingers. Note that (a) shows a soldered pair of bars; (b) shows a cavity with the bars inside, and ready for testing.

Dielectric constant measured under bias: data and conclusions. A possibility to use the operating point at the zero dc bias was investigated. In a series of tests, bias was increased abruptly as e.g. shown in Fig. 42 (top), kept at its value for a while, and brought back to zero. Fig. 42 shows just one example from many obtained in these tests, but the behavior seen in this plot was typical across all samples. One observes a sudden change in the dielectric constant of a sample when the bias jumps up or down, and a subsequent relaxation process of the order of minutes. These long relaxation times to which the ferroelectric, metal-coated ceramic is prone when the bias suddenly grows from zero to some non-zero value (or vice versa) suggest that choosing zero bias as the operating point is not favorable because it will deliver an unnecessary strain on the phase feedback loop to compensate for these slow apparent relaxation times in dielectric constant, which are equivalent to slow drifts in the signal phase. The nature of this apparent long relaxation time is currently unknown, but is expected to be studied by Euclid TechLabs during their own SBIR-supported materials development project. 
Omega-P, Inc.

Phase II proposal submitted following Phase I DoE SBIR grant DE-SC0006229

in response to DoE SBIR/STTR 2011 Solicitation Topic 44a

Nuclear Physics Accelerator Technology, Materials and Components for Radio Frequency Devices

\section{FAST FERROELECTRIC L-BAND TUNER FOR SUPERCONDUCTING CAVITIES}
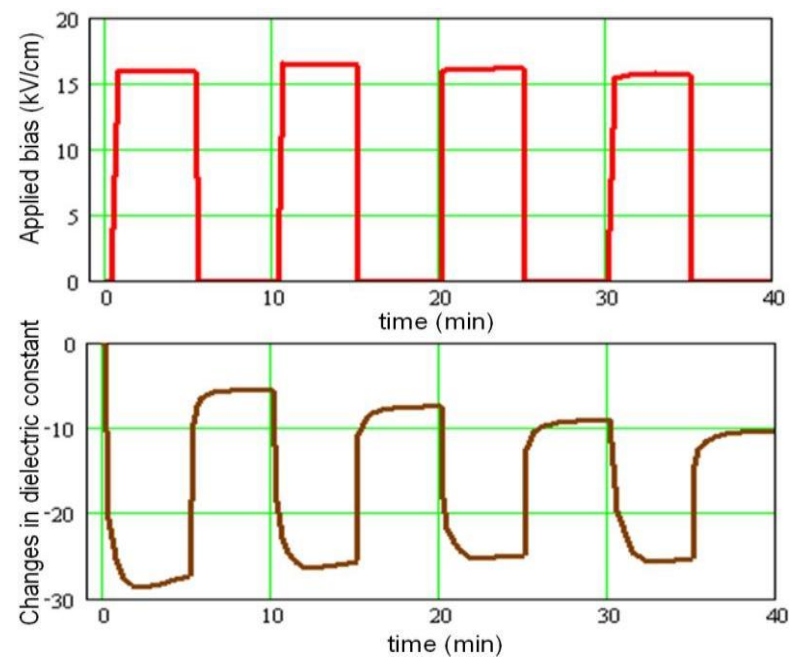

Fig. 42. Changes in dielectric constant of the short bars when the bias is swept from 0 to $\sim 16 \mathrm{kV} / \mathrm{cm}$.

Fortunately, it was found that if one increases the operating point voltage (and never going below it), that helps considerably to reduce relaxation effects. This is shown in the data of Fig. 43.
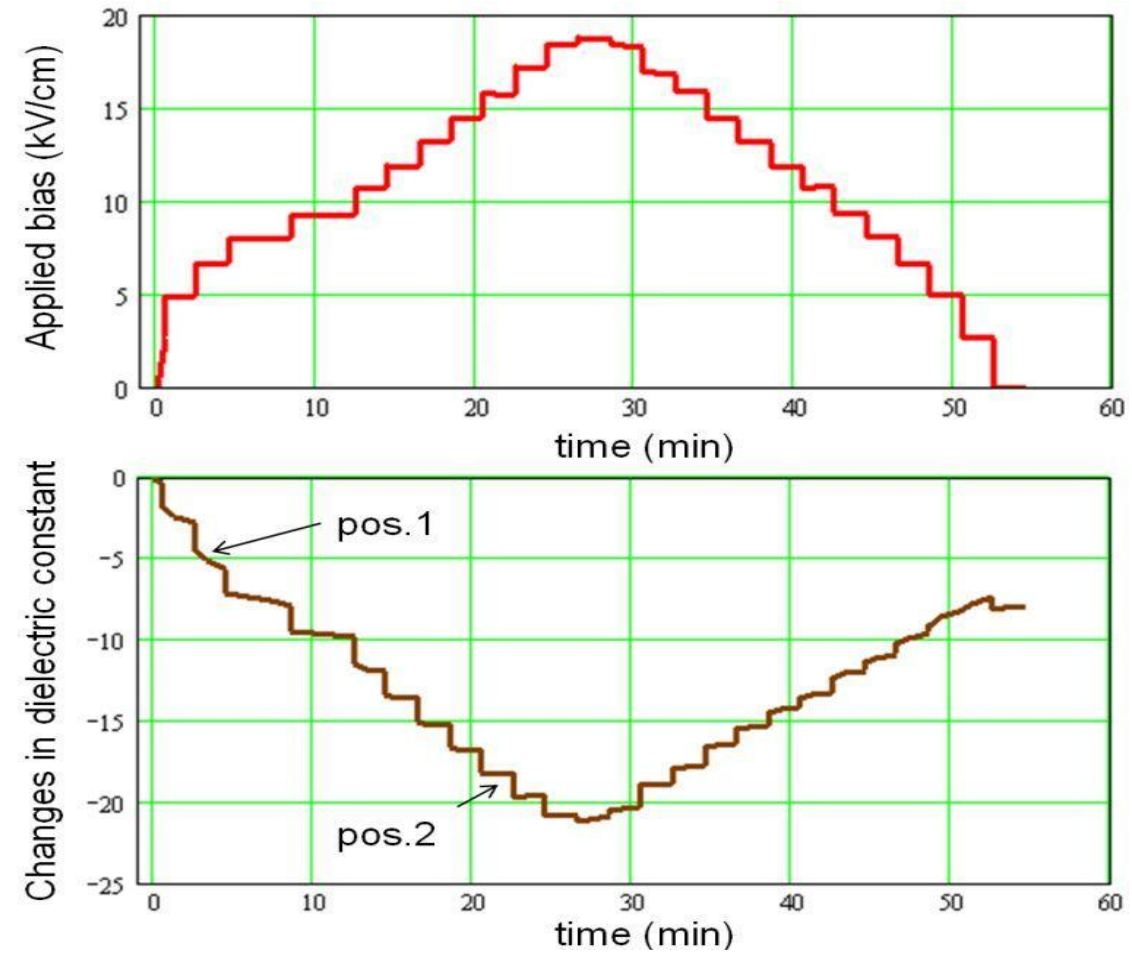

Fig. 43. Example of changes in dielectric constant when the bias is swept in a step-like manner starting from $0 \mathrm{kV} / \mathrm{cm}$. 
Omega-P, Inc.

Phase II proposal submitted following Phase I DoE SBIR grant DE-SC0006229 in response to DoE SBIR/STTR 2011 Solicitation Topic 44a

Nuclear Physics Accelerator Technology, Materials and Components for Radio Frequency Devices

FAST FERROELECTRIC L-BAND TUNER FOR SUPERCONDUCTING CAVITIES

A series of test were performed where the bias was changed in a small increments in the manner demonstrated in Fig. 43 (top). Clearly, above $15 \mathrm{kV} / \mathrm{cm}$, the relaxation effects are greatly diminished (compare e.g. pos. 1 with pos.2). It is also clearly seen that if one starts at a bias of 15 $\mathrm{kV} / \mathrm{cm}$ (or above), then increases the bias and then comes back to the $15 \mathrm{kV} / \mathrm{cm}$ bias again, the value of dielectric constant is nearly the same, as opposed to the case when one tries to start (and come back) to any bias voltages below $15 \mathrm{kV} / \mathrm{cm}$. This is in a sound agreement with other research on ferroelectric materials, albeit not in a bulk shape but a form of thin films [16].

To further test this assumption, we started with the samples subjected to high enough bias for a prolonged period of time (several hours), reduced the bias in a step-like manner, and brought it back while recording the changes in the dielectric constant. An example is given by Fig. 44, from which one sees again that with operating bias voltages above $15 \mathrm{kV} / \mathrm{cm}$ (or even $20 \mathrm{kV} / \mathrm{cm}$ ) will not pose exceptional strain on the phase feedback loop to provide compensation for the relaxation-like changes in dielectric constant, which are equivalent to slow drift in the signal phase. One sees in Fig. 44 that the relaxations are greatly diminished when the bias is above 15 $\mathrm{kV} / \mathrm{cm}$ (e.g. pos. 1 or pos.2) as compared to when it is below $15 \mathrm{kV} / \mathrm{cm}$ (e.g. pos. 3 and 4)
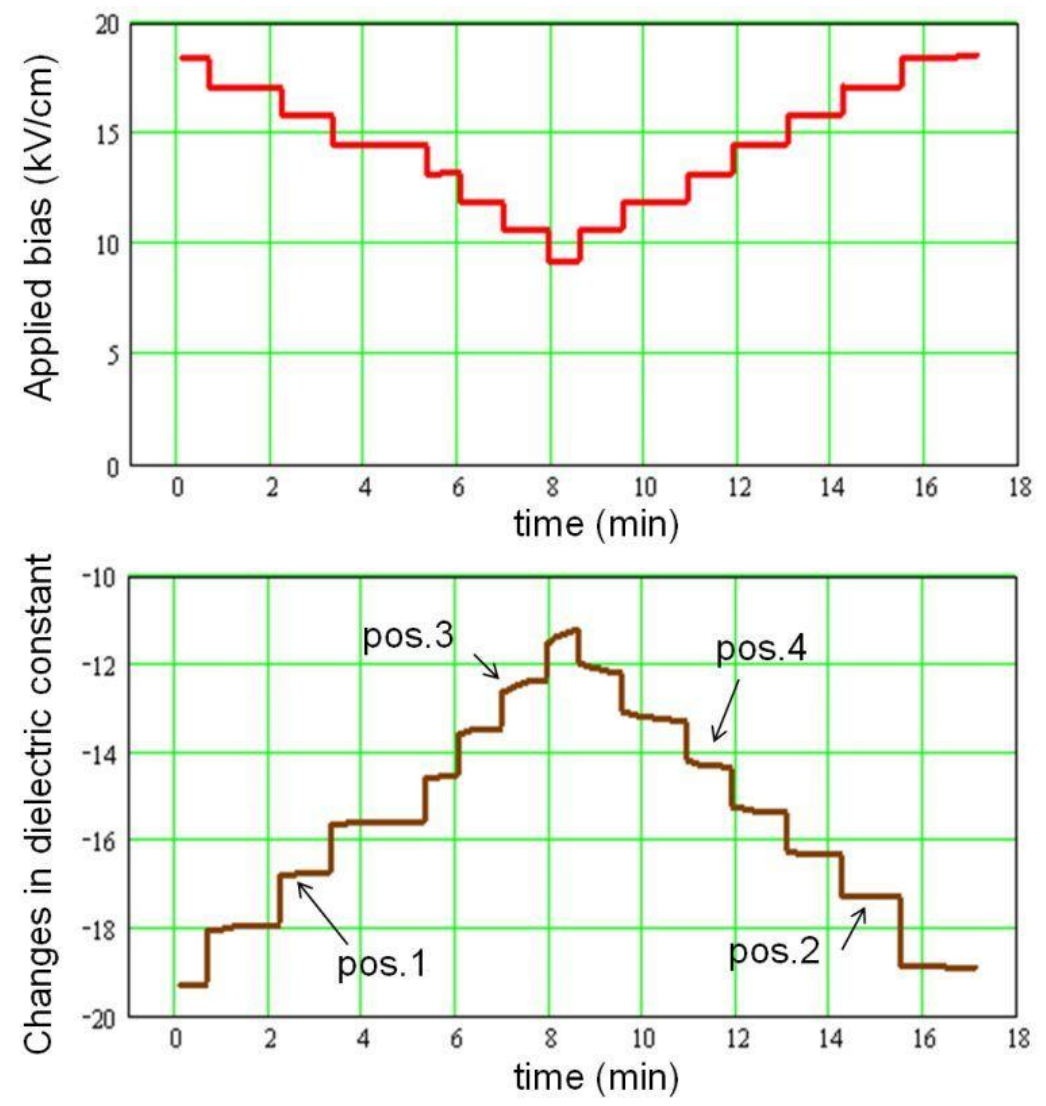

Fig. 44. Example of changes in dielectric constant when the bias is swept in a step-like manner starting from $\sim 18.5 \mathrm{kV} / \mathrm{cm}$ down to $\sim 10 \mathrm{kV} / \mathrm{cm}$ and back. 
Omega-P, Inc.

Phase II proposal submitted following Phase I DoE SBIR grant DE-SC0006229

in response to DoE SBIR/STTR 2011 Solicitation Topic 44a

Nuclear Physics Accelerator Technology, Materials and Components for Radio Frequency Devices

FAST FERROELECTRIC L-BAND TUNER FOR SUPERCONDUCTING CAVITIES

The described tests were limited by the capabilities of the available high-voltage power supply; but tests at higher bias voltages are planned.

From these measurements, one concludes that, with ferroelectric samples prepared and metalized as were these samples, one should operate at biases exceeding $15-20 \mathrm{kV} / \mathrm{cm}$. The operation point at $\sim 30 \mathrm{kV} / \mathrm{cm}$ can be perhaps recommended, with sweeping the bias voltage down to $20 \mathrm{kV} / \mathrm{cm}$ to change the dielectric constant to control the signal phase.

The fast response time of the metal-coated ceramic is to compensate for abrupt changes in phase with a speed sufficient to suppress phase instabilities an SRF cavity. The slow changes in dielectric constant are equivalent to slow changes in phase (phase drifts) which will present in the system anyway. Feedback loops to compensate for slow phase changes are well known, will be employed, but these are not a subject of present project.

Dielectric loss tangent under bias: data and conclusions. Measurements of loss tangent indicate that changes occur when the bias changes. As with the dielectric constant, it is unfavorable to have a scheme that has its operating point at zero bias. The changes in loss tangent are not observed to be small. An example is given in Fig. 45.

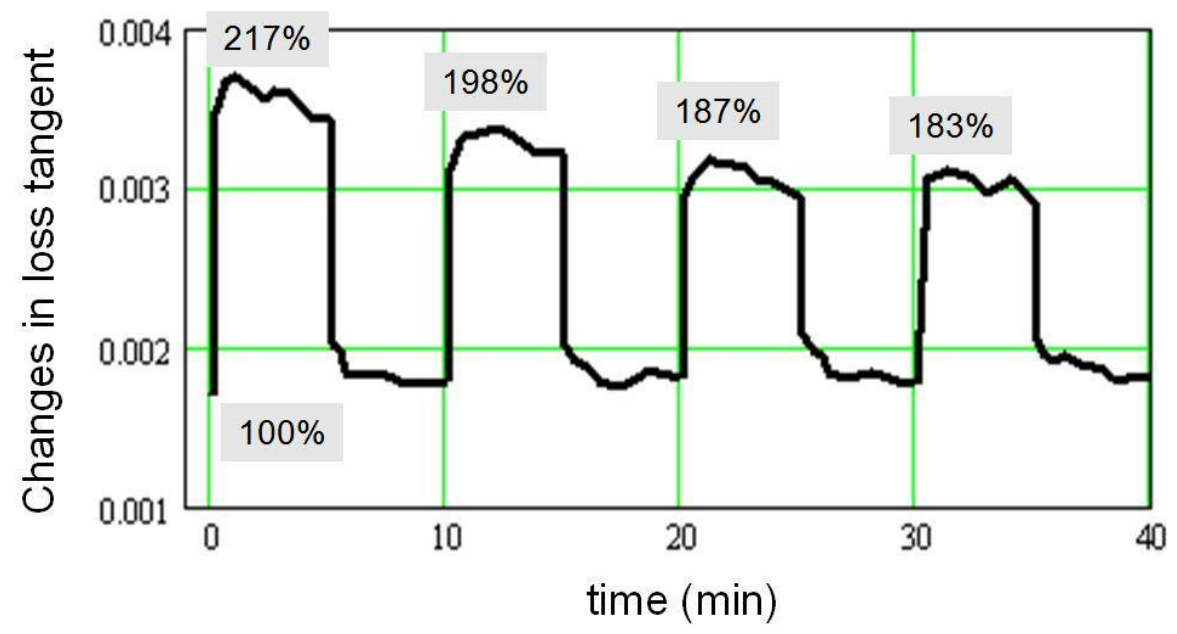

Fig. 45. Example of measured changes in dielectric constant when the bias is swept in a steplike manner starting from $0 \mathrm{kV} / \mathrm{cm}$, using the bias profile as shown in Fig. 42.

An improved scenario is possible when one biases the ferroelectric, metal-coated ceramic at some value exceeding $15 \mathrm{kV} / \mathrm{cm}$ (perhaps an initial bias values as large as $20-30 \mathrm{kV} / \mathrm{cm}$ can be recommended [17]). This is demonstrated in Fig. 46.

While the dielectric constant changes enough [see Fig. 44] to provide the required tunability range, the loss tangent changes by a factor of up to $150 \%$ [see the right-hand side of Fig. 46]. This effect is to be a subject of further study by Euclid TechLabs during their own SBIRsupported materials development project. 
Omega-P, Inc.

Phase II proposal submitted following Phase I DoE SBIR grant DE-SC0006229 in response to DoE SBIR/STTR 2011 Solicitation Topic 44a

Nuclear Physics Accelerator Technology, Materials and Components for Radio Frequency Devices

FAST FERROELECTRIC L-BAND TUNER FOR SUPERCONDUCTING CAVITIES

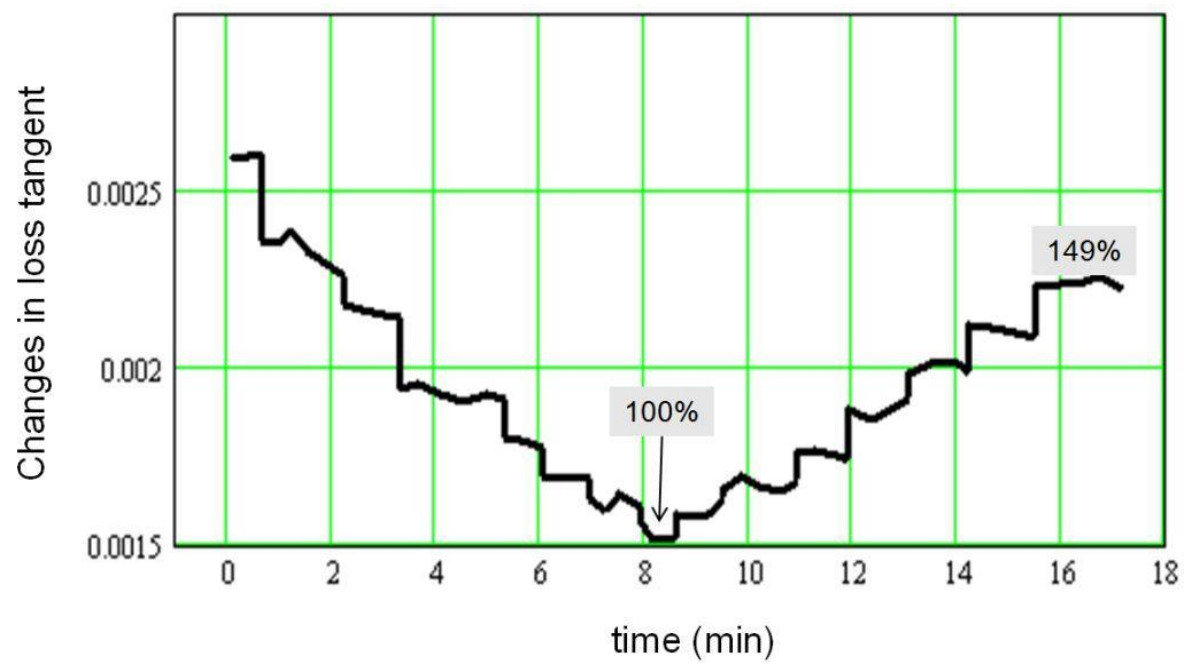

Fig. 46. Example of measured changes in dielectric constant when the bias is swept in a steplike manner starting from $\sim 18.5 \mathrm{kV} / \mathrm{cm}$ down to $10 \mathrm{kV} / \mathrm{cm}$ and back to $18.5 \mathrm{kV} / \mathrm{cm}$, following the bias profile as shown in Fig. 44.

To reduce the dependence of dielectric constant and loss tangent on bias, it is concluded that further development of the metalizing procedures themselves is necessary. One clue that the metallization is the culprit is the fact that the intrinsic response time of the ferroelectric material is in the range of $30 \mathrm{~ns}$, as shown in Fig. 37, when no metallization is present. Possible ways to achieve this include: (1) trying metal layers based not on $\mathrm{Cr}$ (chrome), but Pt (platinum); (2) varying the temperature of a coated sample to reduce the oxygen depletion of the surface of the BST ceramic; (3) trying to perform the Pt-layer deposition in a slightly pressurized chambers with a fraction of oxygen present not high enough to oxidize Pt, but enough to prevent oxygen depletion of the ceramic surface. The oxygen depletion leads to vacancies in the near surface layer, and this layer behaves as a semiconductor that introduces additional capacitance (with the value that depends on the bias) leading to the aforementioned relaxation phenomena. These steps are expected to be followed by Euclid TechLabs during their own SBIR-supported materials development project.

However, while it is highly desired to improve the properties of the ferroelectric ceramic, including its metal coating to reduce the dependence of the loss tangent on the bias, it is not required for first prototype demonstrations of the Omega-P design for the phase shifter module described in this proposal. Fortunately, with already available metal-coated ferroelectric ceramic it is possible to develop a phase shifter that should be able to demonstrate operation with a SC cavity showing a savings in drive power in the range of 5-10, as compared with the case of no phase shifter. 


\section{Omega-P, Inc.}

Phase II proposal submitted following Phase I DoE SBIR grant DE-SC0006229

in response to DoE SBIR/STTR 2011 Solicitation Topic 44a

Nuclear Physics Accelerator Technology, Materials and Components for Radio Frequency Devices

FAST FERROELECTRIC L-BAND TUNER FOR SUPERCONDUCTING CAVITIES

\section{References}

1. T. Zwart, D. Cheever, W.S. Graves, D. Wang, A. Zolfaghari, "Optimal RF System For Lightly Loaded Superconducting Structures", Proceedings of the 2004 FEL Conference, pp. 542-545; see also web.elettra.trieste.it/fel2004/proceedings/papers/TUPOS57 /TUPOS57.PDF

2. S. Simrock., G. Petrosyan, A. Facco, V. Zvyagintsev, S. Andreoli, and R. Paparella, "First Demonstration of Microphonic Control of a Superconducting Cavity with a Fast Piezoelectric Tuner", PAC2003, Portland, May 11-16, 2003, p.470.

3. M. Liepe and S. Belomestnykh, "Microphonics Detuning in the $500 \mathrm{MHz}$ Supercondicting CESR Cavities," PAC2003, Portland, May 11-16, 2003, pp.1326-1328.

4. Y. Kang, M. Tiefenback, and P. Chevtsov, "Reactive RF Tuning for Compensation of a Detuned Accelerating Cavity," Linac 2002, Gyeongju, Korea, 2002, pp.733-735.

5. V.P. Yakovlev, S.Yu. Kazakov, and J.L. Hirshfield, "1.3 GHz Electrically-Controlled Fast Ferroelectric Tuner," EPAC2006, Edinburgh, June 26-30, 2006, pp. 487-489.

6. Y. Kang, J. Wilson, M. McCartney, S-H. Kim, M. Champion, R. Kustom, B. Chase, "Development of TEM High Power RF Vector Modulators", CWHAP06, May 1-4, 2006, Argonne, IL

7. D. Valuch, H. Frischholz, J. Tückmantel, and C. Weil, "First Results With A Fast Phase And Amplitude Modulator For High Power RF Applications," EPAC2004, pp. 959-961.

8. D. Horan, and E. Cherbak, "Fast-Ferrite Tuner Operation On A 352-MHz Single-Cell RF Cavity At The Advanced Photon Source," PAC2003, pp. 1177-1179.

9. B. Foster, I. Gonin, T. Khabiboulline, A. Makarov, N. Solyak, I. Terechkine, and D. Wildman, "High Power Phase Shifter," PAC2005, PAC2005, Knoxville, May 16-20, 2005, p. 3123-3125.

10. M. Liepe and S. Belomestnykh,"RF Parameters and Field Stability Requirements for the Cornell ERL Prototype", PAC2003, Portland, May 11-16, 2003, pp.1326-1328.

11. I. Ben-Zvi, "The ERL High-Energy Cooler for RHIC", EPAC2006, Edinburgh, 2006, pp. 940-944. See also R. Calaga, "SRF Cavities for High Current ERLs," BNL, January 23, 2006, http://www.bnl.gov/cad/ecooling/docs/PDF/MAC_01_06/calaga.pdf

12. S. Holmes, "Project X: Status, Strategy, Meeting Goals," Project X Collaboration Meeting, Fermilab, September 8, 2010, http://projectx-docdb.fnal.gov/cgibin/RetrieveFile?docid=721\&version=2\&filename $=$ PX $\% 20$ Collabortion $\% 20$ Meeting $\% 2$ 02010_SDH.pdf

13. S. Belomestnykh, "RF system issues due to pulsed beam in ILC DR," IWLC, CERN, October, 2010, http://ilcagenda.linearcollider.org/getFile.py/access?contribId=202 \&sessionId=77\&resId=3\&materialId=slides\& confId $=4507$

14. S. Yu. Kazakov, S. V. Shchelkunov, V. P. Yakovlev, A. Kanareykin, E. Nenasheva, and J. L. Hirshfield, Phys. Rev. ST Accel. Beams 13, 113501 (2010) "Fast Ferroelectric Phase Shifter Design for ERLs Proc. of ERL09, Ithaca, New York, USA, June 8-12, 2009, p. 67; web-link: http://accelconf.web.cern.ch/AccelConf/ERL2009/papers/wg317. pdf 


\section{Omega-P, Inc.}

Phase II proposal submitted following Phase I DoE SBIR grant DE-SC0006229 in response to DoE SBIR/STTR 2011 Solicitation Topic 44a

Nuclear Physics Accelerator Technology, Materials and Components for Radio Frequency Devices

\section{FAST FERROELECTRIC L-BAND TUNER FOR SUPERCONDUCTING CAVITIES}

15. S. Yu. Kazakov, S. V. Shchelkunov, V. P. Yakovlev, A. Kanareykin, E. Nenasheva, and J. L. Hirshfield, Phys. Rev. ST Accel. Beams 13, 113501 (2010)

16. A.B. Kozyrev, M.M. Gaidukov, A.G. Gagarin, A.G. Altynnikov, S.V. Razimuv, A.V. Tumarkin, "Influence of Metal-Ferroeletric Contacts on the Space Charge Formation in Ferroelectric Thin Film Capacitors",Tech. Phys. Lett, 2009, Vol.35. No.7, pp.585-588, and refs therein. ISSN (printed) 1063-7850, ISSN (eletronic) 1090-6533

17. V.A. Volpyas, A.G. Gagarin, A.B. Kozyrev, A.G. Altynnikov, "Distribution of Nonlinear Charge Carriers in a Nonlinear Thin-Film Capacitor",Tech. Phys. Lett, 2007, Vol.33. No.10, pp.844-846, and refs therein.ISSN (printed) 1063-7850, ISSN (eletronic) 10906533.

\section{Relevant Publications and Patents}

1. S. Yu. Kazakov, S. V. Shchelkunov, V. P. Yakovlev, A. Kanareykin, E. Nenasheva, and J. L. Hirshfield, "Fast Ferroelectric Phase Shifter Design for ERLs," in Proceedings of ERL09, Ithaca, New York, USA, June 8-12, 2009, p. 67; web-link: http://accelconf.web.cern.ch/AccelConf/ERL2009/papers/wg317. pdf

2. S. Yu. Kazakov, S. V. Shchelkunov, V. P. Yakovlev, A. Kanareykin, E. Nenasheva, and J. L. Hirshfield, "Fast ferroelectric phase shifters for energy recovery linacs," Phys. Rev. ST Accel. Beams 13, 113501 (2010).

3. United States Patent \#7,786,675 B2, issued August 31, 2010, entitled "Fast Ferroelectric Phase Shift Controller for Accelerator Cavities." Inventors are Vyacheslav P. Yakovlev, Sergey Y. Kazakov, and Jay L. Hirshfield. Assignee: Omega-P, Inc.

4. United States Provisional Patent Application \#61/619,741, filed April 3, 2012, entitled "Ferroelectric Tuner." Inventors are Sergey Y. Kazakov, Vyacheslav P. Yakovlev, and Sergey Shchelkunov. Assignee: Omega-P, Inc. This provisional application contains improvements to the designs protected under the above patent, including those described in this report. 